\title{
1 A toolbox to profile immunometabolism tested in macrophages
}

2 Sanne G. S. Verberk ${ }^{1,4}$, Kyra E. de Goede ${ }^{1,4}$, Friederike S. Gorki ${ }^{1,2}$, Xanthe A.M.H. van

3 Dierendonck ${ }^{1}$, Rafael J. Argüello ${ }^{3}$, Jan Van den Bossche ${ }^{1, *}$

41 Department of Molecular Cell Biology and Immunology, Amsterdam Cardiovascular

5 Sciences, Amsterdam Gastroenterology Endocrinology Metabolism, Amsterdam Institute for

6 Infection and Immunity, Cancer Centre Amsterdam, Amsterdam UMC, Vrije Universiteit

7 Amsterdam, Amsterdam, Netherlands

82 Institute of Innate Immunity, University Hospital Bonn, University of Bonn, Bonn 53127,

9 Germany

$10{ }^{3}$ Aix Marseille Univ, CNRS, INSERM, CIML, Centre d'Immunologie de Marseille-Luminy, 11 Marseille, France

$12{ }^{4}$ These authors contributed equally (really)

$13 \quad{ }^{*}$ Corresponding author: j.vandenbossche@amsterdamumc.nl

\section{Summary}

Macrophages are highly plastic immune cells that can adopt several activation states. Often, macrophages are stimulated in vitro with lipopolysaccharide (LPS) to elicit pro-inflammatory macrophages or interleukin (IL)-4 to obtain a homeostatic phenotype. Fundamental to these functional activation states is the regulation of cellular metabolic processes. The metabolic alterations underlying LPS- and IL-4-induced phenotypes have been described thoroughly with several -omics techniques. However, methods allowing for time- and cost-effective screening of cellular metabolism are rather limited. Here, we establish a comprehensive toolbox to assess cellular metabolism in a semi-high-throughput 96-well-plate-based format. We validated the approach on LPS- and IL-4-activated mouse and human macrophages by measuring extracellular flux, SCENITH, mitochondrial mass and membrane potential, glucose and lipid uptake, and mitochondrial substrate utilization. By discussing the experienced strengths, limitations and complementarities of the distinct techniques, we guide readers for efficient application of the toolbox in their immune subsets of interest.

Keywords: immunometabolism, macrophages, toolbox, semi-high-throughput, metabolism

\section{Motivation}


In the last decade, it has become increasingly clear that cellular metabolism affects and even drives alterations in immune cell function. This has sparked an expansion of (immuno)metabolic studies. However, the different techniques available may seem daunting to researchers new to the field of immunometabolism and can be very time-consuming and costly. We therefore established an immunometabolic toolbox based on a 96-well-plate format and illustrate different techniques on mouse and human macrophages. Next to established methods such as extracellular flux analysis, the toolbox includes the new method SCENITH and substrate-coated plates which have not been previously applied to in vitro macrophages. We further discuss advantages and limitations of each technique to guide readers to effectively use the toolbox in their own research setting.

\section{Introduction}

Macrophages are innate immune cells that reside in tissues or differentiate from circulating monocytes and regulate acute inflammatory responses and tissue homeostasis (Wynn et al., 2013). While they can adopt a whole spectrum of activation states, most research focusses on lipopolysaccharide (LPS)- and interleukin (IL)-4-induced activation states. LPS-induced macrophages (classically activated macrophages, M[LPS]) produce high levels of proinflammatory cytokines and show increased surface expression of (co)stimulatory immune activation markers. Conversely, IL-4-induced macrophages (alternatively activated macrophages, M[IL-4]) upregulate a different set of surface markers and enzymes involved in repair and homeostasis (Murray et al., 2014).

Metabolic rewiring resides at the core of phenotypic polarization and has been shown to direct immune responses (Jha et al., 2015). Upon stimulation with LPS, macrophages increase metabolic flux through glycolysis and the pentose phosphate pathway (PPP) which in turn fuels reactive oxygen species (ROS) production and nitric oxide (NO) synthesis (Van den Bossche et al., 2017, Baardman et al., 2018). M[LPS] macrophages also display a disruption of the tricarboxylic acid (TCA) cycle at two points: isocitrate dehydrogenase (IDH) and succinate dehydrogenase (SDH) (Jha et al., 2015). The LPS-induced downregulation of IDH results in the shunting of (iso)citrate towards synthesis of aconitate and subsequent accumulation of anti-microbial itaconate, mediated by the immunoresponsive gene 1 encoded enzyme IRG1 (Lampropoulou et al., 2016). As a result of a blunted SDH activity, succinate accumulates and functions as an immunoregulatory metabolite in macrophages that directs the immune response via hypoxia-inducible factor 1 alpha (HIF1 $\alpha$ ) and other mechanisms (Mills et al., 2016, Harber et al., 2020). In sharp contrast with M[LPS], IL-4-stimulated macrophages are characterized by an intact TCA cycle, increased arginase-1 (Arg1) activity and fatty acid oxidation (FAO) (Van den Bossche et al., 2017). 
As metabolism dictates functional responses in macrophages and other immune cells, there is substantial potential in generating targeted therapeutics that combat chronic inflammatory disorders and cancer (Lim et al., 2020, Makowski et al., 2020). In the study of new therapeutic metabolic interventions lies the importance of measuring cellular metabolism in a time- and cost-effective manner. Currently, a commonly used method is extracellular flux analysis, which measures extracellular acidification rates (ECAR) and oxygen consumption rates (OCR) as markers for glycolysis and mitochondrial oxidative phosphorylation (OXPHOS), respectively (Van den Bossche et al., 2015). This technique contributed to the key concept that M[LPS] macrophages are more glycolytic, whereas M[IL-4] macrophages show higher OXPHOS and FAO (Van den Bossche et al., 2016, Jha et al., 2015). While the method gives a proper overview of core metabolic pathways, it does not reveal the regulation and activation of more specific cellular metabolic pathways or the direct uptake of nutrients from the environment. A combination of several -omics techniques can explain delicate changes in cellular metabolism. As such, metabolomics analysis measures the abundance of metabolites and can reveal metabolic changes dictated by increased production or decreased substrate usage (Jha et al., 2015, Rattigan et al., 2018). Also, (single-cell) RNA-sequencing (transcriptomics) shows regulation on gene expression level, and aids in evaluating the transcriptional activity in all metabolic pathways (Jha et al., 2015, Artyomov and Van den Bossche, 2020). However, RNAsequencing neither allows the assessment of post-translational modifications that may dictate the function of metabolic enzymes, nor of metabolic enzyme activity. While new methods are arising that permit the measurement of metabolic enzyme abundance and activation by flow cytometry (Hartmann et al., 2021, Ahl et al., 2020, Levine et al., 2021), these are relatively expensive in terms of time and costs, resulting in the inclusion of only a limited number of samples or experimental conditions. Methods allowing for an effective screening of metabolic pathway activity are therefore needed to facilitate drug and inhibitor screens.

Here, we provide a comprehensive set of methods as a toolbox that allows measuring multiple aspects of immune cell metabolism in a 96-well-plate-based format. First, we performed NO production and arginase-activity measurements to quickly show metabolic alterations as a preliminary readout tool. Next, we compare extracellular flux analysis with a recently developed method for measuring single-cell metabolism. Additionally, we measure fluorescent readouts for mitochondrial mass, mitochondrial membrane potential, and glucose and fatty acid uptake. Lastly, we also propose a comprehensive protocol to measure specific substrate usage in permeabilized and intact cells. The methods are applied to the well-described LPS- and IL-4induced mouse and human macrophages providing the tools for low-key and semi-highthroughput immunometabolic research that can be extended to different immune cells. 


\section{Results}

103

104

105

106

107

108

109

110

111

112

113

114

115

116

117

118

119

120

121

122

123

124

125

126

127

128

129

130

131

132

133

134

135

Overview of semi-high-throughput techniques encompassed in the immunometabolic toolbox

We provided a toolbox to interrogate immunometabolism in a semi-high-throughput manner by combining extracellular flux analysis with metabolic analysis of distinct cell subsets using SCENITH (Arguello et al., 2020). We further extended the analyses by measuring the uptake of fluorescent probes both in an imaging multi-mode plate reader and by flow cytometry and by investigating fuel preference between macrophage activation states by measuring the specific substrate utilization (Figure 1).

Extracellular flux analysis validates metabolic remodeling in LPS- and IL-4-activated macrophages

Mouse bone marrow-derived macrophages (BMDMs) and human monocyte-derived macrophages (HMDMs) were left untreated or were stimulated with LPS or IL-4 (Figure 2A). While HMDMs do not show NO production or detectable arginase activity, BMDMs displayed an increased production of NO upon LPS-activation and increased arginase activity upon activation with IL-4 (Figure 2B (BMDMs), data not shown (HMDMs)). Extracellular flux analysis on BMDMs displayed upregulated basal oxygen consumption (Supplementary Figure $1 \mathrm{~A}$ ) and maximal respiration following IL-4 treatment as expected, while LPS-activated macrophages showed a trend towards decreased maximal respiration (Figure $2 \mathrm{C}$ ). These parameters were not significantly altered in HMDMs (Figure 2D). Unexpectedly, LPS-stimulation increased basal respiration both in mouse and human macrophages. Based on ECAR measurements, BMDMs showed the representative upregulation of glycolysis and glycolytic capacity upon LPS-, and to a lesser extent, upon IL-4-stimulation (Figure 2E, Supplementary Figure 1A). While LPSactivated HMDMs exhibited significantly elevated glycolysis (Supplementary Figure 1B), glycolytic capacity was not increased in HMDMs for either stimulation (Figure 2F). Normalization of ECAR and OCR rates was performed using fluorescent imaging of macrophages stained with cell-permeable Hoechst to calculate cell counts, as has been implemented previously (Little et al., 2020, Janssen et al., 2021) (Supplementary Figure 1C). While normalization decreased outliers as shown for glycolytic capacity and maximal respiration, it did not fundamentally alter the data (Supplementary Figure 1D,E). Furthermore, normalization decreased the variation between multiple wells in a single experiment as indicated by reduced standard deviation of both ECAR and OCR-based parameters (Supplementary Figure 1F). Therefore, normalization of extracellular flux data with Hoechst dye injection and subsequent quantification of cell counts by fluorescent imaging is effective in reducing variation within experiments. 
Next, we examined the SCENITH technology which can profile metabolism of distinct cell subsets (Arguello et al., 2020). This technique is based on protein synthesis as a reliable alternative readout for ATP production through metabolic flux, determining the effect of metabolic inhibitors on puromycin incorporation during protein translation by flow cytometry. We assessed mouse and human macrophages with SCENITH and investigated its compliance with extracellular flux analysis.

144 Overall, while a high geometric mean fluorescent intensity (gMFI) of puromycin was detected for control samples indicating a high level of overall protein synthesis in BMDMs, IL-4 led to even increased levels of protein synthesis (Figure $3 \mathrm{~A}$ ). As expected, the gMFI of puromycin was highly dependent on the addition of metabolic inhibitors, without affecting cell viability in BMDMs (Figure 3A, Supplementary Figure 2A). On the other hand, addition of Deoxy-DGlucose (DG) for 30 minutes slightly affected viability in LPS-activated HMDMs, indicating that caution is warranted when interpreting these data without using a viability dye (Supplementary Figure 2A,B). Puromycin gMFI after addition of inhibitors allowed for the calculation of several metabolic parameters: glucose and mitochondrial dependency were calculated as the proportion of protein synthesis dependent on glucose oxidation and OXPHOS, respectively (Figure 3C). Furthermore, glycolytic capacity and fatty acid and/or amino acid oxidation (FAO/AAO) capacity indicate the maximum potential of cells to sustain protein synthesis when OXPHOS and glucose oxidation are inhibited, respectively (Arguello et al., 2020). Addition of DG almost completely abolished the signal in all mouse macrophage stimulations examined, indicating overall high glucose dependency (Figure 3A, D). M[LPS] relied solely on glucose as fuel, while IL-4-treated BMDMs retained at least some capacity for FAO/AAO (Figure 3D). Conversely, oligomycin (O) decreased protein synthesis in naïve and M[IL-4], but barely affected this readout in M[LPS], revealing a distinct reliance on OXPHOS in the different activation states (Figure 3A, D). This was also reflected in the derived metabolic parameters that indicate decreased mitochondrial dependence but high glycolytic capacity of M[LPS] (Figure 3D). For BMDMs, glycolytic capacity as captured by SCENITH correlated significantly with the same parameter as assessed by extracellular flux analysis (Figure 3F).

In HMDMs, very similar metabolic changes upon macrophage activation were found compared with BMDMs. However, HMDMs relied much more on mitochondrial oxidation as evidenced by an increased effect of the mitochondrial inhibitor oligomycin on puromycin gMFI (Figure 3B). Consistently, mitochondrial dependence was higher in naïve and LPS-activated HMDMs compared to their mouse counterparts, accompanied by a lower glycolytic capacity and higher 
capacity for FAO and/or AAO (Figure 3E). Similar to BMDMs, glycolytic capacity as measured with SCENITH correlated well with extracellular flux analysis for HMDMs (Figure 3G).

173

We further confirmed macrophage activation by combining SCENITH with typical activation markers, indicating successful activation of M[LPS] and M[IL-4] (Supplementary Figure 2C-F). To exploit the capacity of SCENITH to study metabolism of distinct subsets within a pool of cells, we performed tSNE dimensionality reduction and FlowSOM single-cell clustering (Van Gassen et al., 2015) on naive and LPS-activated BMDMs with Oligomycin-mediated inhibition of mitochondrial puromycin production (Figure $3 \mathrm{H}$ ). Interestingly, M[LPS] showed a heterogenous activation defining two clusters upon LPS-activation (clusters 3 and 4) as indicated by differing CD40 and iNOS-expression (Figure 3H, Supplementary Figure 2G). Interestingly, puromycin also showed a heterogenous expression across these clusters, indicating that homogenous activation results in different metabolic activation. Since iNOS displayed the biggest difference between clusters 3 and 4 (Supplementary Figure 2G), we analyzed mitochondrial dependency and glycolytic capacity in iNOS Low and iNOS High cell subsets (Figure $3 \mathrm{I}$ ). The iNOS ${ }^{\text {High }}$ subset showed larger LPS-induced changes such as decreased mitochondrial dependency and increased glycolytic capacity, as compared to iNOS Low cells (Figure 3J). In contrast, in IL-4-stimulated BMDMs such distinction was not accompanied by changes in puromycin levels, and therefore did not result in changes in metabolic parameters (data not shown). The single-cell clustering analysis illustrates the capacity of SCENITH to capture heterogeneous responses of cells to a stimulus. Moreover, this allowed us to reveal a more activated metabolic phenotype than what was previously observed using bulk analysis.

Measuring incorporation of fluorescent nutrient analogs by multi-mode reader and flow cytometry yields complementary information to extracellular flux analysis and SCENITH

We further extended metabolic measurements by investigating the uptake of fluorescent metabolic dyes at single-cell level both by multi-mode reader and flow cytometry. Assessment by multi-mode reader showed fluorescent signal of the glucose analog 2NB-DG, the mitochondrial stain MitoTracker Green, the mitochondrial membrane potential-dependent TMRM and the fatty acid BODIPY C16 from cells (Figure 4A). Since the multi-mode reader did not reveal alterations in mean fluorescence per cell between different activation states (Supplementary Figure 3), we additionally validated fluorescent signal by flow cytometry. In line with multi-mode reader data, mitochondrial mass (MitoTracker Green) and mitochondrial membrane potential (TMRM) did not show major alterations upon macrophage activation (Figure 4B,C). Since mitochondrial mass was shown to be correlated with reduced maximal respiration in macrophages and T cells (van der Windt et al., 2012, Baardman et al., 2018), we 
checked the correlation of MitoTracker Green signal and maximal respiration rate as measured

208 by extracellular flux analysis. Similarly, since an increase in membrane potential indicates a 209 leakage of protons from the electron transport chain that can be used for reverse electron 210 transport (Zorova et al., 2018), we checked the correlation of TMRM and the extracellular flux 211 parameter proton leak. However, no correlation was found between either mitochondrial mass 212 and maximal respiration nor between mitochondrial membrane potential and proton leak 213 (Figure 4B,C).

214 In contrast, assessment of 2NB-DG signal with flow cytometry revealed an elevated uptake of 215 2NB-DG in LPS- and IL-4-stimulated macrophages, which correlated well with extracellular 216 flux analysis-determined glycolysis of BMDMs (Figure 4D,E). Interestingly, also the uptake of 217 BODIPY C16 was increased in M[LPS] and M[IL-4] for both mouse and human macrophages. 218 BODIPY C16 signal significantly correlated with basal respiration as measured by extracellular 219 flux analysis in HMDMs and neared a significant correlation in BMDMs (Figure 4D,E). While 220 fluorescent metabolic probes reveal activation-specific signals, the uptake of nutrient analogs 221 does not reveal the fate of the nutrient within the cell nor the dependence on the nutrient. 222 Nutrients can be metabolized or can be stored and accumulated in different compartments of 223 cells. By measuring glucose and fatty acid uptake, one can reveal whether glucose or fatty 224 acid uptake resides at the source of glycolytic and respiration rates. Thereby, the uptake levels 225 of fluorescent probes aids the interpretation of functional metabolic analyses such as extracellular flux analysis or SCENITH.

227 Analysis of biochemical substrate utilization rates gives additional insight in fuel preference and metabolic enzyme activity

229 While the combination of extracellular flux analysis, SCENITH and the measurement of 230 fluorescent probes gives a marked insight in metabolic functioning, the actual fuel preference 231 of cells in a certain activation state remains largely unknown. To gather an enhanced view on 232 mitochondrial enzyme activities in LPS- and IL-4-activated macrophages, we assessed 233 mitochondrial substrate utilization rates with permeabilized cells in substrate-coated plates. A 234 redox dye allowed for the quantification of substrate utilization by measuring absorbance over 235 time.

236 Mitochondrial substrate conversion by permeabilized cells was measured during a course of 5 237 hours (Supplementary Figure 3A, B). Substrates that require malate for mitochondrial 238 conversion were supplemented with $100 \mu \mathrm{M}$ Malic acid (sparker malate) (Gerhardt et al., 239 1995). Maximum oxidation rates indicate the enzymatic capacity to oxidize the specific 240 substrates, and revealed differential regulation of mitochondrial substrate oxidation upon 241 activation with LPS or IL-4 (Figure 5A, B). Oxidation of TCA cycle intermediates displayed a 
242 marked decrease in both LPS- and IL-4-activated BMDMs (Figure 5C). Whereas LPS

243 downregulated the oxidation of all TCA cycle intermediates, IL-4-activation only affected a

244 selection of substrates downstream of cis-aconitic acid (Figure 5C). While the uptake of 245 fluorescent analog long-chain (16C) fatty acids was markedly increased upon LPS when 246 measured by flow cytometry (see Figure 4D), this was not paralleled by increased 247 mitochondrial oxidation of short-, medium-, or long chain-fatty acids, but rather decreased 248 oxidation of $8 \mathrm{C}$-fatty acids (Figure $5 \mathrm{E}$ ).

249 Similarly, human LPS-activated macrophages displayed a marked decrease in the oxidation 250 of substrates of the TCA cycle, whereas differences between IL-4-activated and naïve 251 macrophages were less pronounced (Figure 5B). While no statistical significance was reached 252 in HMDMs, some TCA cycle intermediates like citrate, succinate and malate showed a trend 253 towards decreased maximum oxidation rate with M[LPS], with no changes found with M[IL-4] 254 (Figure 5E, F). Since permeabilization of cells does not allow for the measurement of cytosolic 255 metabolic pathways, the MitoPlates S-1 did not measure alterations in glycolysis but provided 256 increased insight into mitochondrial remodeling upon LPS- or IL-4-stimulation.

257 Glycolysis is one of the main differentially regulated pathways in LPS- and IL-4-activated 258 macrophages as assessed by extracellular flux, SCENITH and 2NB-DG uptake. We further 259 investigated the regulation of glycolysis in BMDMs using PM-M01 Biolog plates that allow for 260 the measurement of substrate oxidation in intact cells. D-glucose was the most upregulated 261 utilized substrate in both M[LPS] and M[IL-4] (Supplementary Figure 3B). Whilst significant 262 differences were observed in the oxidation of other carbohydrates upon macrophage 263 activation, such as maltose and fructose, the biological relevance remains undefined. 264 Interestingly, the maximum rate of D-glucose oxidation correlated well with glycolysis as 265 determined by extracellular flux analysis and with 2NB-DG uptake (Supplementary Figure 3C), 266 indicating that assessing glucose oxidation with a redox dye in intact cells is a relevant 267 additional tool to study glycolysis.

268 Together, the techniques assessed here investigate metabolism from different angles and all 269 contribute to a deeper understanding of metabolic phenotypes. In the next section, strengths 270 and limitations will be considered alongside a discussion of the results. 


\section{Discussion}

272 Immune cell function is highly controlled by their metabolism. We here illustrate the use of 273 several techniques for metabolic profiling and present an in-depth view of mitochondrial 274 function with a 96-well plate-based semi-high-throughput method on well-described mouse 275 and human macrophages activated with LPS or IL-4. This toolbox allows for time- and cost276 efficient screening of candidate drugs before moving towards more advanced metabolic 277 evaluation. It can also easily be extended to ex vivo samples and other (immune) cells.

\section{Guide to choose the right technique in your circumstances}

279 In recent years, immunologic research has increasingly focused on metabolic remodeling in immune cells obtained after in vitro activation or from complex in vivo samples. To get an initial idea about metabolic alterations in immune cells, a first selection can be made based on colorimetric assays on cells, lysates, or their supernatants (Figure 6). Glucose and lactate measurements allow for the detection of glucose usage and lactate production by the cells. Additionally, consistently with our results, mouse, but not human, macrophages show increased nitric oxide production upon activation with LPS and increased arginase activity after stimulation with IL-4 (Thomas and Mattila, 2014, Gross et al., 2014, Cameron et al., 1990, Schneemann et al., 1993). As a final pre-screen, cells can be analyzed for mitochondrial activity by an MTT assay that is based on the conversion of tetrazolium salt MTT $(3-(4,5-$ dimethylthiazol-2-yl)-2,5-diphenyl tetrazolium bromide) by the TCA cycle enzyme succinate dehydrogenase.

As a subsequent step, our toolbox allows for an extended overview of different metabolic activation profiles upon immune cell activation (Figure 6). The toolbox can be followed up by more costly and in-depth analyses like metabolomics, ${ }^{13} \mathrm{C}$-based fluxomics, and RNAsequencing, as commonly applied for bulk analyses. More complex in vivo samples consisting of a heterogeneous pool of cells can be studied by flow and mass cytometry-based metabolic profiling (including SCENITH), single-cell RNA-sequencing, or spatial metabolomics and immunohistochemistry for metabolic enzymes (Figure 6) ((Artyomov and Van den Bossche, 2020) for recent review). Since these techniques all have their advantages and disadvantages, they should be used in their specific, most suitable applications and combined with complementary techniques.

\section{Extracellular flux analysis for bulk analysis of cells}

Extracellular flux analysis is currently the predominantly used metabolic profiling technique, giving it the status of 'golden standard'. The injection of several inhibitors and metabolic compounds during an extracellular flux assay allows for determining the contribution of a 
specific metabolic pathway to acidification and oxygen consumption. In accordance with earlier literature, we here found increased glycolysis in LPS-induced BMDMs (Van den Bossche et al., 2015, Lauterbach et al., 2019). Parallel to BMDMs, we also found increased glycolysis in human M[LPS], consistent with an earlier proteomic study of LPS-activated HMDMs (RealesCalderon et al., 2014). Decreased glycolysis has also been reported upon LPS-stimulation in HMDMs (Vijayan et al., 2019), and these contrasting results may be explained by differences in the timing of cellular activation and subsequent measurement (Lauterbach et al., 2019). In line with this, time-dependent metabolic changes have been shown previously for BMDMs and are likely to occur in HMDMs as well (Thomas and Mattila, 2014, Lauterbach et al., 2019).

Whereas LPS-stimulation in BMDMs showed decreased maximal respiration in our results, an even augmented decrease in maximal respiration was shown in the past for BMDMs and results at least partly from LPS-induced NO production (Van den Bossche et al., 2016, Van den Bossche et al., 2015). Contrarily to BMDMs, HMDMs produce no NO due to epigenetic silencing of NOS2 (Gross et al., 2014), and may therefore not decrease maximal respiration (Vijayan et al., 2019). This is consistent with our results for NO production and maximal respiration in HMDMs. Additionally, we observed a significant increase in basal respiration in LPS-activated human macrophages and less for mouse macrophages. Overall, this indicates that human and mouse macrophages behave slightly different in terms of metabolic remodeling.

In the current study, IL-4-induced BMDMs upregulated maximal respiration compared to naïve BMDMs as has been reported before (Van den Bossche et al., 2016). Originally, this was thought to mainly result from increased $\mathrm{FAO}$, which was believed to be crucial for alternative macrophage activation (Vats et al., 2006, Huang et al., 2014a). However, experiments with carnitine palmitoyltransferase 1 and 2 (Cpt1/2) knockout mice indicated that FAO is largely dispensable for IL-4-induced macrophage activation (Van den Bossche and van der Windt, 2018, Nomura et al., 2016, Divakaruni et al., 2018). The same was also found for HMDMs (Namgaladze and Brune, 2014). Glycolysis was then believed crucial for IL-4-macrophage activation (Huang et al., 2016), but this was later nuanced as glycolysis is not required for activation as long as OXPHOS is intact (Wang et al., 2018). Our finding that both mouse and human IL-4-activated macrophages upregulate glycolysis, is consistent with this. A previous study did recognize the high reliance of M[IL-4] on glucose, but did not see induction of basal glycolysis (Rodríguez-Prados et al., 2010). This discrepancy may result from the use of different macrophage types, BMDMs in our setting versus RAW cells and peritoneal macrophages in earlier studies (Rodríguez-Prados et al., 2010). 
While extracellular flux analysis already provided multiple parameters in the currently applied study set-up, outlining the buffer capacity of the assay medium can even indicate specific

341 glycolytic and mitochondrial contribution to ATP production (Mookerjee et al., 2017).

342 Additionally, dependency on main fuels can be determined by the combination of extracellular 343 flux assays with injections of inhibitors such as etomoxir (fatty acids), BPTES (pyruvate) and 344 UK5099 (glutamine) (Voss et al., 2021). However, caution is warranted as not all inhibitors are specific (Divakaruni et al., 2018). Also, another set of personally preferred inhibitors or compounds can give rise to additional metabolic data for an even more comprehensive overview of cellular metabolism, for example to assess different components of the electron transport chain (Salabei et al., 2014).

On the other hand, extracellular flux analysis also comes with certain disadvantages limiting its applications. Whereas extremely useful for homogeneous samples of which many cells are present (such as in vitro differentiated and stimulated cells), only the core metabolic pathways that directly result in $\mathrm{H}^{+}$production or oxygen consumption are presented. Additionally, analysis of complex in vivo samples such as tumor tissue is much more challenging. The latter includes many distinct cell populations which cannot be separately assessed with extracellular flux analysis alone. To gather metabolic parameters per cell type with extracellular flux analysis, cells of interest need to be sorted before analysis. Both cell sorting and possible associated incubation in cell culture media may affect the metabolic state of cells (Voss et al., 2021, Binek et al., 2019, Llufrio et al., 2018). Generally, large cell numbers are required for extracellular flux analysis, but new developments now allow to measure rare cell populations in smaller and specifically designed plates. However, with this approach, less replicates can be included.

\section{SCENITH for metabolic phenotyping of distinct cell subsets}

To tackle the measurement of cell subsets in more complex samples, Argüello et al. (Arguello et al., 2020) recently provided a simple flow cytometry-based way of investigating cellular metabolism. As this technique can be combined with lineage and activation markers, SCENITH is excellently equipped to enable simultaneous metabolic investigation of distinct cell populations, including rare cell populations. Here, we used the method and found a high dependence of BMDMs and HMDMs on glucose. Both human and mouse LPS- and IL-4induced macrophages showed an elevated glycolytic capacity compared to naïve macrophages, which is in line with extracellular flux data. In general, BMDMs and HMDMs revealed similar patterns of metabolic dependence, although HMDMs relied more on

372 OXPHOS. Overall, SCENITH-derived glycolytic capacity correlated well with extracellular flux data. 
374 In our experiments, we used the method to validate metabolic characterization of 375 homogenously-activated in vitro samples and illustrated its use in a panel with different 376 activation markers. Interestingly, SCENITH enabled the identification of metabolic differences 377 in two subpopulations of LPS-activated BMDMs distinguished mostly by iNOS expression. The 378 heterogeneity in these populations could not have been found with extracellular flux analysis 379 alone, since bulk analysis would have provided an average of these populations. The 380 possibility of applying SCENITH in different flow cytometry panels allows for the analysis of 381 even more heterogeneous samples. Whereas metabolic, differentiation and activation markers 382 can be subjective to changes by inflammatory activation, lineage markers like F4/80, CD3 or 383 CD19 generally allow for proper gating independent of inflammatory activation. SCENITH is 384 therefore an interesting additive in large immune panels that also permits single-cell clustering. 385 However, it should be noted that single-cell clustering analysis in combination with SCENITH should be performed with caution. As the metabolic parameters are calculated from inhibited samples treated in parallel rather than from one single sample, one cannot substantially show the metabolic parameters calculated for each single cell in the analysis. SCENITH in combination with larger flow cytometry-based immune panels therefore rather shows metabolic alterations in cell subsets and not in single cells per se. To overcome this, we here clustered cells from samples that were all treated with oligomycin to reveal a heterogeneous expression of puromycin and thereby a heterogeneous mitochondrial dependency and glycolytic capacity. SCENITH may further provide novel insights in combination with large metabolic flow cytometry panels. However, the stability of metabolic markers upon treatment with inhibitors should be validated beforehand and additionally, this approach quickly becomes much more costly, since each sample needs to be split and measured in parallel in order to accommodate the different inhibitors.

Fluorescent metabolic probes for the analysis of bulk and single-cell metabolic features

399

400

401

402

403

404

405

406

407

408

409

The use of a multi-mode reader improves extracellular flux measurements by providing a quick and easy way of cell count-based normalization. It further allows for the imaging of cells stained with fluorescent probes in a 96-well-plate-based manner, but the resolution of our machine did not allow for the determination of differences in signal between distinct macrophage activation states in our experiments. Therefore, we here determined fluorescence by flow cytometry, which is better equipped for quantification of data but lacks an option for visual information, which can be important in other fluorescent measurements such as phagocytosis assays. Here, we showed a significant correlation of 2NB-DG uptake with glycolysis as measured by extracellular flux analysis and glucose usage by BMDMs as determined in a PM-M01 substrate utilization analysis. This indicates that the transported glucose into the cell is used in glycolysis and results, at least partly, in lactic acid secretion. However, care needs to be taken when 
assessing glucose uptake solely by fluorescent labeling as it was previously shown that 2NBDG staining yielded substantially different results compared to glucose transport assays and 2NB-DG uptake was not impeded by glucose transport inhibitors in T cells (Sinclair et al., 2020).

Additionally, we revealed a significant correlation of BODIPY C16 uptake with basal respiration. Interestingly, BODIPY C16 uptake was increased in LPS-activated BMDMs and HMDMs, but was not reflected in an increased FAO/AAO capacity as measured with SCENITH or increased fatty acid utilization as determined with mitochondrial substrate utilization in MitoPlates S-1. This indicates that while LPS-induced macrophages take up fatty acids, the cells rather store them (e.g. in lipid droplets) than using them to fuel metabolic processes, as has been reported previously (Feingold et al., 2012, Huang et al., 2014b). Next to 2NB-DG and BODIPY C16, we here measured MitoTracker Green for mitochondrial mass estimation and TMRM to assess mitochondrial membrane potential. While activation of LPS and IL-4 did not show differences in signal for these markers, the markers can be relevant for other research questions. For example, we have previously shown that a reduced maximal respiration was (partly) explained by reduced mitochondrial mass as measured with MitoTracker Green (Baardman et al., 2018).

Next to the fluorescent probes measured here, a range of other fluorescent dyes and analyses exist that can be included to analyze metabolic alterations or cellular adjustments in response to specific treatments. Examples of other probes are mitoSOX for the measurement of mitochondrial ROS or propidium iodide for the analysis of cell cycle distribution. Additionally, a confocal microscope or multi-mode reader with higher resolution can be used to reveal visual differences in subcellular localization or mitochondrial fragmentation (Little et al., 2020).

While the uptake of 2NB-DG and BODIPY C16 by macrophages did correlate with extracellular flux parameters, specific fuel preference remains unknown and requires further analysis.

During the assessment of substrate oxidation, substrate-coated plates provided more detailed insight compared to the use of fluorescent dyes or by applying inhibitors in extracellular flux analysis. The analysis of substrate utilization comes with some major advantages. Firstly, many specific substrates can be assessed simultaneously, allowing for relatively easy screening of fuel preferences. Secondly, since it is possible to measure sequential substrates,

441 the induction of metabolic pathways like glycolysis and OXPHOS, but also fatty acid and amino

442 acid metabolism can be investigated in greater detail than with the methods mentioned before. 
443 Lastly, substrate utilization gives an enhanced insight in alterations of enzymatic capacities

444 upon cell activation.

445 Although the method may yield new insights in metabolic rewiring, assessment of 446 mitochondrial substrate utilization specifically requires permeabilization of the cell, resulting in 447 a more artificial system than metabolic assessment of intact cells. By permeabilization, cells 448 lose their ability to regulate cellular substrate uptake and mitochondria are thereby provided 449 with ad libitum access to the substrates. As enzyme rates are partly determined by substrate 450 concentration, enzymes will increase their activity upon unlimited substrate availability. These 451 enzymes might then behave differently than under physiological circumstances. Furthermore, 452 in a biological setting, substrates may not be able to reach the cellular compartment where the 453 metabolizing enzymes are located. Loss of compartmentalization in permeabilized cells can 454 lead to enhanced substrate oxidation which may reflect a theoretical potential, but not the 455 physiological metabolic pathway.

456 This point is further illustrated with our finding that permeabilized IL-4-activated BMDMs 457 showed a decreased utilization of multiple TCA cycle intermediates, while they show elevated oxygen consumption with extracellular flux analyses. This apparent discrepancy may be explained by differences in the readouts examined, as mitochondrial substrate utilization indicates activity of TCA cycle enzymes specifically. Alternatively, a decreased utilization may also arise from the permeabilization itself, as extracellular flux analysis assesses whole cell metabolism as compared to mitochondrial metabolism. Additionally, TCA cycle substrate utilization was unaltered in IL-4-stimulated intact BMDMs when compared to naive BMDMs, as measured in PM-M01 plates. The permeabilization itself removes potentially relevant cytoplasmic metabolic enzymes that may convert accumulating TCA cycle intermediates in intact cells for further use. An example is the cytoplasmic conversion of citrate to acetyl-CoA (Lauterbach et al., 2019), which usually aids in further metabolic processing and signaling and thereby may influence mitochondrial metabolism.

During the measurement of intact cells in PM-M01 substrate-coated plates we found significant differences in the utilization of specific sugars upon activation of BMDMs with IL-4 or LPS. Although D-glucose uptake correlated well with other readouts, the relevance of most other sugars remains unknown. Since PM-M01 assays were mainly developed to investigate 473 bacterial metabolism, the differentially regulated utilization of these sugars has not been 474 described in the context of macrophage metabolism before. Although still of high interest and 475 worthy of further investigation, the results can possibly also be attributed to the method being 476 rather artificial in supplying solely one substrate. Therefore, researchers should take care that 477 optional additional substrates are equally relevant to mammalian cells in their analyses. 
This substrate utilization assay is suited to assess homogeneous in vitro cultured cell subsets rather than complex tissue samples. It is helpful when screening metabolic pathways of interest

480 (i.e., identified by previous metabolic experiments such as extracellular flux analysis, SCENITH 481 or uptake of fluorescent probes) for altered substrate utilization, indicating modified enzyme activity. This substrate assay permits the narrowing of focus onto a few substrates or their metabolizing enzymes, which may subsequently be studied in greater detail and even integrated with transcriptomics or metabolomics data.

\section{Beyond the toolbox}

486 Transcriptomics and metabolomics measurements can additionally provide valuable input for 487 the assessment of metabolic changes. However, they show a static measurement of cells at a 488 specific time-point, and seemingly discrepant results may be obtained as a result of measurements providing data on different levels of regulation. For example, posttranscriptional or post-translational modifications are not included in transcriptomics data, and differences in gene expression do not always correlate to active translation into protein or alternatively to enzyme activity. For a further discussion of (single-cell) transcriptomics, metabolomics and their integration (Jha et al., 2015), other techniques to study cellular metabolism such as cytometry-based metabolic panels (Hartmann et al., 2021, Ahl et al., 2020, Levine et al., 2021), and more detailed considerations on how best to assess metabolism and single-cell options, we refer the reader to recent reviews (Artyomov and Van den Bossche, 2020, Voss et al., 2021). Whereas these methods will also greatly enhance the view of metabolic alterations upon treatments with a compound of interest, they also require more specialized machines, and will greatly complicate data analysis.

500 In the metabolic analysis of complex in vivo samples, the spatial and temporal knowledge of metabolic processes are of major additional value (Artyomov and Van den Bossche, 2020, Murphy and O'Neill, 2020). Therefore, imaging-based techniques (Miller et al., 2017, Palmer et al., 2017) such as GeoMX (Farren et al., 2020) or MIBI-TOF (Keren et al., 2019, Hartmann and Bendall, 2020) are developed that combine transcriptomics, metabolic and functional readouts with spatial information, respectively. Also, approaches that assess subcellular metabolism are important since metabolites accumulate to different extents in diverse subcellular locations and may affect (signaling) targets differently. This was for example indicated for acyl intermediates and their ability to modify target proteins (Murphy and O'Neill, 2020). Additionally, to prove whether a metabolic change is cause or consequence of phenotypic alterations, it is desirable to assess temporal aspects. This can be implemented by

511 repeated ${ }^{13} \mathrm{C}$-metabolomic flux measurements in combination with phenotypic assessment or the addition of pseudo-time in single-cell RNA-sequencing. By combining space and time in 
513 spatiotemporal approaches in immunometabolic research, discrepancies in the literature may

514 be resolved. Lastly, causality needs to be demonstrated by targeting metabolic enzymes with

515 genetic or pharmacological tools as described previously in more detail (Voss et al., 2021).

516

\section{Acknowledgements}

518 We thank Sam de Decker, Enrico Tatti and Barry Bochner for supplying all materials to perform 519 the Biolog MitoPlate S-1 and PM-M01 assays, their help with setting up the assays, with 520 interpreting the results and their helpful comments on the manuscript. We further thank Amber 521 Eggert for optimizing the Hoechst cell counting protocol on the Cytation 5 and Karl Harber for 522 proofreading the manuscript. JVdB received a VENI grant from ZonMW (91615052) and a 523 Netherlands Heart Foundation junior postdoctoral grant (2013T003) and senior fellowship 524 (2017T048). Additionally, JVdB received an Enw-Klein-1 grant from NOW 525 (OCENW.KLEIN.268) and a consortia grant from European Research Area network on 526 Cardiovascular Diseases (ERA-CVD 2019T108). Lastly, we acknowledge support from Cancer 527 Center Amsterdam.

\section{Author contributions}

529 SV and KdG contributed equally to this work. Conceptualization: JVdB, SV, KdG. Methodology: 530 SV, KdG, FG, RA. Formal analysis: SV, KdG. Investigation: SV, KdG, FG, XvD. Visualization: 531 SV, KdG. Writing-review\&editing: SV, KdG, FG, XvD, RA, JVdB, Funding acquisition: JVdB. 532 All authors read and approved the final manuscript.

\section{Declarations of interest}

534 The authors declare no competing interests. There are restrictions to the commercial use of 535 SCENITH due to a pending patent application (PCT/EP2020/060486). 
538 Figure 1: Overview of semi-high-throughput techniques encompassed in the metabolic toolbox. (A)

539 Extracellular flux analysis by the Seahorse Bioanalyzer that measures acidification to estimate glycolysis and 540 oxygen consumption as a measurement for OXPHOS in response to metabolic inhibitors. (B) The flow cytometry541 based metabolic profiling technique SCENITH (Arguello et al., 2020), that measures changes in the level of 542 translation in response to inhibitors as a measure for cellular metabolism. (C) Fluorescent measurement of the 543 uptake of several metabolic dyes by the imaging multi-mode plate reader and flow cytometry. (D) Measurements of 544 substrate utilization using permeabilized and intact cells with substrate-coated plates and the measurement of the 545 absorbance of a redox dye based on the production of $\mathrm{NAD}(\mathrm{P}) \mathrm{H}$ and $\mathrm{FADH}_{2}$.

Figure 2: Extracellular flux analyses of BMDMs and HMDMs yield metabolic profiles of macrophages after LPS and IL-4-activation. (A) BMDMs and HMDMs were left untreated (shown in black), or stimulated with either LPS (shown in red) or IL-4 (shown in blue) for $24 \mathrm{~h}$. (B) Levels of NO in supernatants of naive and LPS-stimulated BMDMs and arginase activity in naive and IL-4-activated BMDMs. (C, D) Normalized OCR, with injections of glucose, oligomycin, FCCP and Antimycin A/Rotenone/Hoechst and maximal respiration for BMDMs (C) and HMDMs (D). (E,F) Normalized ECAR with same injections as for OCR and glycolytic capacity for BMDMs (E) and HMDMs $(F)$. For $N O, N=5$, for arginase $N=3$ mice from 5 or 3 different experiments. For kinetic flux measurements, $\mathrm{N}=9$ mice or $\mathrm{N}=10$ donors were included with 3-4 technical replicates each. Per mouse/donor, the median value of technical replicates was used to calculate an overall mean. Kinetic data are depicted as mean \pm SEM of mice/donors. Extracellular flux parameters represent median value for each mouse/donor as calculated from 3-4 technical replicates.

Figure 3: Metabolic analysis of BMDMs and HMDMs with SCENITH reveals typical regulation by LPS and IL-4 activation. (A, B) gMFI of puromycin across samples treated with different inhibitors for BMDMs (A) and HMDMs (B). (C) Calculations of metabolic SCENITH parameters based on puromycin gMFI. (D, E) SCENITH parameters as calculated for mouse $(D)$ and human $(E)$ macrophages. $(F, G)$ Correlation of glycolytic capacity as measured with extracellular flux analysis with glycolytic capacity as measured with SCENITH for BMDMs (F) and HMDMs (G). (H) tSNE dimensionality reduction and clustering analysis of naive and M[LPS] BMDMs by FlowSOM and a heatmap showing the expression of activation markers and puromycin. (I) Gating of iNOS Low and iNOS High for LPS-activated BMDMs. (J) SCENITH parameters calculated for iNOS Low and iNOS High populations. Data are shown as mean \pm SEM. Each dot marks a separate mouse $(\mathrm{N}=2)$ or donor $(\mathrm{N}=2-3)$.

Figure 4: Uptake of fluorescent probes provides additional insight into macrophage metabolism. (A) Representative images of staining by fluorescent dyes and uptake of fluorescent nutrient analogs as assessed by multi-mode reader. Scale bar represents $200 \mu \mathrm{m}$. (B,C) Fluorescent intensity of MitoTracker Green and TMRM analysis as examined by flow cytometry and correlations with parameters of extracellular flux analysis in BMDMs (B) and HMDMs (C). (D,E) Fluorescent intensity of 2NB-DG and BODIPY C16 uptake by BMDMs (D) and HMDMs (E) as examined by flow cytometry, correlated with relevant parameters of extracellular flux analysis. Data are shown as mean \pm SEM. For graphs of fluorescent probes, each dot marks a separate mouse $(N=2-3)$ or donor $(\mathrm{N}=3)$. $\triangle \mathrm{MFI}$ was calculated as MFI (median fluorescent intensity) of sample - MFI of unstained control.

Figure 5: Assessment of mitochondrial substrate oxidation provides insight in specific mitochondrial enzymatic activity by activated macrophages. (A, B) Scatterplot of maximum rate in the timespan of 1-4h of substrate oxidation by LPS- and IL-4-activated compared to naïve BMDMs (A) and HMDMs (B). Substrates belonging to amino acid metabolism, TCA cycle, glycolysis and fatty acid metabolism are depicted in yellow, light green, dark green and grey, respectively. Significantly different substrates are marked with $\left(^{*}\right)$ and substrates supplemented with sparker malate with \#. (C,D) Maximum rate of the oxidation of specific TCA-cycle substrates in permeablized BMDMs (C) and HMDMs (D). (E,F) Oxidation of short-, medium-, and long-chain fatty acids in BMDMs $(E)$ and HMDMs $(F)$. Data are shown as mean \pm SEM. For scatterplots, a mean maximum rate was calculated for all mice or donors. In $B, C, D$ and $F$, each dot marks a separate mouse $(N=4-5)$ or donor $(N=4-5)$.

Figure 6: A guide to direct researchers from simple screening towards complex measurement of immunometabolic alterations 


\section{Bone marrow isolation and BMDM culture}

593 Mouse experiments were approved by the Committee for Animal Welfare of the VU University 594 Amsterdam. 8-16 week old male and female C57BI/6 mice were housed in groups of four in 595 SPF conditions at $21^{\circ} \mathrm{C}$ until sacrifice. Bone marrow cells were isolated from femurs and tibias 596 by flushing with PBS. Bone marrow-derived macrophages (BMDMs) were generated by culturing in complete RPMI-1640 (Gibco) containing 25 mM HEPES, 2 mM L-glutamine, 10\%

598 FCS (Gibco), $100 \mathrm{U} / \mathrm{mL}$ penicillin, $100 \mu \mathrm{g} / \mathrm{mL}$ streptomycin (all Gibco), and 15\% L929599 conditioned medium (LCM) for 7 days. Cells were collected and plated for experiments at a 600 density of $1^{*} 10^{6}$ cells per $\mathrm{mL}$ and were either left untreated or stimulated with $100 \mathrm{ng} / \mathrm{mL}$ LPS 601 (Sigma) or $20 \mathrm{ng} / \mathrm{mL}$ IL-4 (Peprotech) for 24 hours.

\section{Monocyte isolation and HMDM culture}

603 Buffy coats $(50 \mathrm{~mL}$ ) were received from Sanquin blood Bank (Amsterdam, Netherlands). 604 PBMCs were isolated with a Ficoll/Lymphoprep gradient (Greiner Biosciences) and careful 605 centrifugation at $800 \mathrm{~g}$ for 30 minutes. Next, monocytes were isolated by applying $120-150 * 10^{6}$ cells on top of a $46 \%$ Percoll $^{\mathrm{TM}}$ (Cytiva) solution followed by careful centrifugation at $2000 \mathrm{rpm}$ for 20 minutes. Monocytes were plated in IMDM medium containing HEPES (Gibco) supplemented with $2 \mathrm{mM} \mathrm{L}$-glutamine, $100 \mathrm{U} / \mathrm{mL}$ penicillin, $100 \mu \mathrm{g} / \mathrm{mL}$ streptomycin (full IMDM medium), and containing 1\% FCS. After settling for one hour, medium was replaced with full IMDM medium with 10\% FCS and $25 \mathrm{ng} / \mathrm{mL}$ M-CSF (Miltenyi) and cells were differentiated for 6 days with a refreshment of M-CSF-supplemented medium after 3 days.

\section{NO production and Arginase activity assay}

NO production was measured by adding Griess reagent $\left(2.5 \% \quad \mathrm{H}_{3} \mathrm{PO}_{4}\right.$ (Merck), $1 \%$ sulfanilamide (Sigma Aldrich), and 0.1\% naphtylene diamide dihydrochloride (Sigma Aldrich) in $\mathrm{H}_{2} \mathrm{O}$ ) to cell supernatants (1:1) and optical density was measured at $540 \mathrm{~nm}$

Arginase activity was determined on cell lysates. Cells were washed with PBS and lysed by incubating for 30 minutes with $100 \mu \mathrm{l} 0.1 \%$ Triton X-100 (Sigma Aldrich), $25 \mathrm{mM}$ Tris- $\mathrm{HCl}(\mathrm{pH}$ 7.5, Roche) supplemented with $1 \times$ protease inhibitor cocktail (Roche). Arginase was activated by adding $3.5 \mu \mathrm{l}$ of $10 \mathrm{mM} \mathrm{MnCl}_{2}$ (Sigma Aldrich) to $10 \mu \mathrm{l}$ sample and incubated at $56^{\circ} \mathrm{C}$ for 10 620 minutes. Next, samples were incubated with $10 \mu \mathrm{l} 0.5 \mathrm{M} \mathrm{L-arginine} \mathrm{(pH} \mathrm{9.7,} \mathrm{Sigma} \mathrm{Aldrich)}$ 621 for 60 minutes at $37^{\circ} \mathrm{C}$. Reaction was stopped by adding $90 \mu \mathrm{l}$ stop solution $\left(96 \% \mathrm{H}_{2} \mathrm{SO}_{4} / 85 \%\right.$ $622 \mathrm{H}_{3} \mathrm{PO}_{4} / \mathrm{H}_{2} \mathrm{O}$ 1:3:7, Merck) and incubated with $4 \mu \mathrm{l} \alpha$-isonitrosopropiophenone (9\%, Sigma 623 Aldrich) for 30 minutes at $95^{\circ} \mathrm{C}$. Samples were left in the dark to cool down to room temperature 
624 until measurement of optical density at $540 \mathrm{~nm}$. Enzymatic activity was calculated by

$625[\text { Urea }]^{*}\left(\right.$ total volume $\left.\left.{ }^{*} 10^{6}\right) /\left(\text { tested volume }{ }^{*} \text { Time (incubated at } 37^{\circ} \mathrm{C}\right)^{*} 1000\right)$.

\section{Extracellular flux analysis}

627 Extracellular flux analysis was performed using the Seahorse XFe-96 Flux Analyzer (Agilent) 628 to examine oxygen consumption (OCR) and extracellular acidification rates (ECAR) as 629 described previously (Van den Bossche et al., 2015). Briefly, BMDMs and HMDMs were plated 630 at a density of $7.5^{*} 10^{5}$ cells per well in XF-96-cell culture plates (Agilent) and stimulated for 24 631 hours with LPS or IL-4, or left untreated. 1 hour prior to the assay, cells were washed and 632 medium was replaced by DMEM (Sigma-Aldrich) without glucose, phenol red, and sodium 633 bicarbonate, supplemented with $5 \mathrm{mM}$ HEPES and $2 \mathrm{mM} \mathrm{L}$-glutamine. The run consisted of 2 634 minutes mixing, 3 minutes measuring and subsequent 4 injections; Glucose (final 635 concentration in well $25 \mathrm{mM}$ ), Oligomycin (O, final concentration $1.5 \mu \mathrm{M}$ ), FCCP (final 636 concentration $1.5 \mu \mathrm{M})$, and antimycin $\mathrm{A}(\mathrm{AA}$, final concentration $2.5 \mu \mathrm{M})$ with rotenone (rot, 637 final concentration $1.25 \mu \mathrm{M}$ ) and Hoechst 33342 (Thermo Fisher) (final concentration $5 \mu \mathrm{g} / \mathrm{mL}$ ). 638 Directly after the run, Hoechst signal was measured on the Cytation 5 Cell Imaging multi-mode 639 reader (BioTek) with a 4X magnification using a $365 \mathrm{~nm}$ LED in combination with an EX377/50 640 EM 447/60 filter cube and cell counts were analyzed using Gen $5^{\mathrm{TM}}$ software. Subsequently, 641 flux rate data was normalized to cell counts and analyzed using Wave software version 642 2.6.0.31 as described previously (Van den Bossche et al., 2015).

\section{SCENITH and Flow cytometry and analysis}

644 SCENITH protocol was performed as described previously (Arguello et al., 2020). Briefly, control or metabolic inhibitors Deoxy-D-glucose (DG, final concentration $100 \mathrm{mM}$ ), oligomycin

$646(\mathrm{O}$, final concentration $1 \mu \mathrm{M})$, combination of $\mathrm{DG}$ and $\mathrm{O}(\mathrm{DGO})$, or Harringtonine $(\mathrm{H}$, final concentration $2 \mathrm{mg} / \mathrm{mL}$ ) as negative control were added to fully differentiated cells and incubated for $15 \mathrm{~min}$ at $37^{\circ} \mathrm{C}$. Subsequently, puromycin (final concentration $10 \mathrm{mg} / \mathrm{mL}$ ) was added without washing and incubated for another $30 \mathrm{~min}$ at $37^{\circ} \mathrm{C}$. After incubation, cells were washed with cold PBS and proceeded with Fc receptor blockade (eBioScience for mouse, BD Biosciences for human cells), fixable viability dye and surface antibody staining in PBS/0.5\% BSA $/ 0.02 \%$ sodium azide (PBA) for $30 \mathrm{~min}$ at $4^{\circ} \mathrm{C}$ in the dark. Cells were then washed, fixed an permeabilized using the FOXP3 fixation and permeabilization kit (eBioScience) according

654 to manufacturer's instructions. For intracellular staining of iNOS, Arg1 and puromycin, cells were incubated for $1 \mathrm{~h}$ at $4^{\circ} \mathrm{C}$ in antibody staining solution in permeabilization buffer. 
659 Data were analyzed using FlowJo (TreeStar, v10) and were compensated using single stains 660 with UltraComp eBeads (ThermoFisher) labeled with the appropriate fluorochrome. Next, cells 661 were gated on FSC-A/SSC-A to gate out debris, then on FSC-A/FSC-H to identify single-cells 662 and lastly on FVD- viable cells.

663 For unbiased tSNE analyses, files were uploaded to the OMIQ online analysis platform 664 (https://omiq.ai/), scaled and subsampled to include 10.000 cells live single cells per file. Next, 665 the tSNE tool set to 1500 iterations, a perplexity of 30 and a theta of 0.5 was used to create 666 tSNE dimensionality reduction. Cells were then clustered using the FlowSOM algorithm using 6675 metaclusters (Van Gassen et al., 2015) and clusters were overlaid on tSNE dimensionality 668 reduction. Clusters were further assessed using the 'Clustered Heatmap' tool in OMIQ to 669 identify discriminating markers between clusters.

670 Vehicle control, DG, O, Harringtonine, puromycin and anti-puromycin antibodies were received 671 as SCENITH kit from (http://www.scenith.com) (Arguello et al., 2020). A complete list of 672 antibodies used can be found in Supplementary Table 1.

673 Fluorescent metabolic dyes

674 Mitochondrial mass was measured using MitoTracker Green (Invitrogen). Mitochondrial 675 membrane potential was measured using Tetramethylrhodamine methyl ester (TMRM, 676 Thermo Fisher) and fatty acid and glucose uptake was measured using BODIPYTM FL $\mathrm{C}_{16}$ 677 (BODIPY, Thermo Fisher) or 2-(N-(7-Nitrobenz-2-oxa-1,3-diazol-4-yl)Amino)-2-Deoxyglucose 678 (2NB-DG, Invitrogen) fluorescent probes. Cells were plated in 96-well black culture plates at a 679 density of $8^{*} 10^{4}$ cells per well. Cells were starved for 2 hours in basal RPMI-medium with or 680 without glucose (for 2NB-DG) and subsequently stained by incubation for 30 minutes in 681 complete RPMI with either 50 nM MitoTracker, 100 nM TMRM, $25 \mu \mathrm{M}$ 2NB-DG or with $1 \mu \mathrm{M}$ BODIPY. Hoechst (final concentration $5 \mu \mathrm{g} / \mathrm{mL}$ ) was added for the last 5 minutes of incubation.

683 Cells were washed with PBS and imaged in $4 \mathrm{X}$ magnification on the Cytation 5 at $37^{\circ} \mathrm{C}$ using 684 a $465 \mathrm{~nm}$ LED in combination with an EX 469/35 EM 525/39 filter cube for MitoTracker Green, 685 BODIPY and 2NB-DG and a 523 nm LED in combination with an EX 531/40 EM 593/40 filter 686 cube for TMRM. Masks were generated around cells positive for the dye of interest using the 687 Gen5 software, validated using positive Hoechst signal and subsequently, fluorescent intensity 688 was calculated per cell.

689 After measurement on the Cytation 5, cells were harvested using ice-cold PBS and 690 immediately acquired on an X20 Fortessa flow cytometer (BD Biosciences) and analyzed as 691 described in the section 'Flow cytometry and analysis'.

\section{Mitochondrial functional substrate assay}


693 Mitochondrial functional substrate assays were performed using MitoPlates S-1 (Biolog). First,

694 cells for mitochondrial functional substrate assay were seeded in a 96 well culture plate at a 695 density of $8^{*} 10^{5}$ cells per well and stimulated for 24 hours with LPS or IL-4, or left untreated. 1 696 hour prior to the assay, $30 \mu \mathrm{l}$ assay buffer with $1 \mathrm{X}$ saponin (Sigma-Aldrich, final concentration $69750 \mu \mathrm{g} / \mathrm{mL}$ ) and redox dye MC was dispensed into the wells of the Mito S-1 plate and incubated 698 at $37^{\circ} \mathrm{C}$. Then, cells were washed once with $100 \mu \mathrm{l}$ assay buffer and subsequently incubated 699 with $35 \mu$ assay buffer with $1 \mathrm{X}$ saponin at room temperature for permeabilization. After 15 700 minute incubation time, permeabilized cells were mixed by pipetting up and down and incubated for another 15 minutes at room temperature. Lastly, $30 \mu \mathrm{l}$ of permeabilized cell suspension in $1 \mathrm{X}$ saponin was transferred to the MitoPlates S-1 plate.

703

704

705

706

707

708

709

710

711

712

713

714

715

716

717

718

719

720

721

722

723

724

Additionally, intact BMDMs were plated as $4 * 10^{4}$ cells per well in $50 \mu \mathrm{l} \mathrm{MC-0}$ Assay Medium into PM-M01 plates (Biolog) to further assess whole-cell substrate usage as opposed to only mitochondrial substrate use. Cells and substrates were pre-incubated for 24 hours at $37^{\circ} \mathrm{C}$ and $5 \% \mathrm{CO}_{2}$, and metabolism was assessed after adding $20 \mu \mathrm{l}$ X Biolog Redox Dye MB.

Color formation in MitoPlates S-1 and PM-M01 plates was measured by the automated platform OmniLog (Biolog) and maximum rates between 1 and 4 hours of analysis were determined using Biolog Data Analysis software version 1.7.1.58.

\section{Statistical analysis}

Data are presented as mean \pm standard error of the mean (SEM) unless specified differently. Statistical significance was analyzed using an ordinary one-way or two-way ANOVA where appropriate followed by Sidak's correction for multiple comparisons in GraphPad Prism software (8.2.1) using paired analysis to minimize effects of mouse/donor variation. For substrate utilization experiments, significant substrates were determined with a Student's tdistribution using Biolog Data Analysis Software. $P$ values $<0.05$ were considered statistically significant indicated by ${ }^{*} p<0.05,{ }^{* *} p<0.01,{ }^{* * *} p<0.001$. The number of mice or donors included in each experiment is indicated in the figure legend. Except for SCENITH, all experiments were performed as at least 2 independent experiments. For NO and Arginase assays, extracellular flux analyses and fluorescent probe uptake, at least 3 technical replicates were included to calculate a mean value to represent a mouse or donor.

\section{Data availability}

All presented data is available from the authors upon reasonable request.

\section{SCENITH materials Availability}


725 Adapted protocols and all the reagents including the panel of inhibitors, puromycin and the

726 monoclonal antibody clone R4743L-E8, conjugated with Alexa Fluor 647 or Alexa Fluor 488

727 (SCENITH kit) are available upon application at http://www.scenith.com/. 


\section{References}

AHL, P. J., HOPKINS, R. A., XIANG, W. W., AU, B., KALIAPERUMAL, N., FAIRHURST, A. M. \& CONNOLLY, J. E. 2020. Met-Flow, a strategy for single-cell metabolic analysis highlights dynamic changes in immune subpopulations. Commun Biol, 3, 305.

ARGUELLO, R. J., COMBES, A. J., CHAR, R., GIGAN, J. P., BAAZIZ, A. I., BOUSIQUOT, E., CAMOSSETO, V., SAMAD, B., TSUI, J., YAN, P., BOISSONNEAU, S., FIGARELLA-BRANGER, D., GATTI, E., TABOURET, E., KRUMMEL, M. F. \& PIERRE, P. 2020. SCENITH: A Flow Cytometry-Based Method to Functionally Profile Energy Metabolism with Single-Cell Resolution. Cell Metab, 32, 1063-1075 e7.

ARTYOMOV, M. N. \& VAN DEN BOSSCHE, J. 2020. Immunometabolism in the Single-Cell Era. Cell Metab, 32, 710-725.

BAARDMAN, J., VERBERK, S. G. S., PRANGE, K. H. M., VAN WEEGHEL, M., VAN DER VELDEN, S., RYAN, D. G., WUST, R. C. I., NEELE, A. E., SPEIJER, D., DENIS, S. W., WITTE, M. E., HOUTKOOPER, R. H., O'NEILL L, A., KNATKO, E. V., DINKOVAKOSTOVA, A. T., LUTGENS, E., DE WINTHER, M. P. J. \& VAN DEN BOSSCHE, J. 2018. A Defective Pentose Phosphate Pathway Reduces Inflammatory Macrophage Responses during Hypercholesterolemia. Cell Rep, 25, 2044-2052 e5.

BINEK, A., ROJO, D., GODZIEN, J., RUPEREZ, F. J., NUNEZ, V., JORGE, I., RICOTE, M., VAZQUEZ, J. \& BARBAS, C. 2019. Flow Cytometry Has a Significant Impact on the Cellular Metabolome. J Proteome Res, 18, 169-181.

CAMERON, M. L., GRANGER, D. L., WEINBERG, J. B., KOZUMBO, W. J. \& KOREN, H. S. 1990. Human alveolar and peritoneal macrophages mediate fungistasis independently of L-arginine oxidation to nitrite or nitrate. Am Rev Respir Dis, 142, 1313-9.

DIVAKARUNI, A. S., HSIEH, W. Y., MINARRIETA, L., DUONG, T. N., KIM, K. K. O., DESOUSA, B. R., ANDREYEV, A. Y., BOWMAN, C. E., CARADONNA, K., DRANKA, B. P., FERRICK, D. A., LIESA, M., STILES, L., ROGERS, G. W., BRAAS, D., CIARALDI, T. P., WOLFGANG, M. J., SPARWASSER, T., BEROD, L., BENSINGER, S. J. \& MURPHY, A. N. 2018. Etomoxir Inhibits Macrophage Polarization by Disrupting CoA Homeostasis. Cell Metab, 28, 490-503 e7.

FARREN, M. R., SAYEGH, L., WARE, M. B., CHEN, H. R., GONG, J., LIANG, Y., KRASINSKAS, A., MAITHEL, S. K., ZAIDI, M., SARMIENTO, J. M., KOOBY, D., PATEL, P., EL-RAYES, B., SHAIB, W. \& LESINSKI, G. B. 2020. Immunologic alterations in the pancreatic cancer microenvironment of patients treated with neoadjuvant chemotherapy and radiotherapy. JCI Insight, 5 .

FEINGOLD, K. R., SHIGENAGA, J. K., KAZEMI, M. R., MCDONALD, C. M., PATZEK, S. M., CROSS, A. S., MOSER, A. \& GRUNFELD, C. 2012. Mechanisms of triglyceride accumulation in activated macrophages. $J$ Leukoc Biol, 92, 829-39.

GERHARDT, B., FISCHER, K. \& MAIER, U. 1995. Effect of palmitoylcarnitine on mitochondrial activities. Planta, 196, 720-726.

GROSS, T. J., KREMENS, K., POWERS, L. S., BRINK, B., KNUTSON, T., DOMANN, F. E., PHILIBERT, R. A., MILHEM, M. M. \& MONICK, M. M. 2014. Epigenetic silencing of the human NOS2 gene: rethinking the role of nitric oxide in human macrophage inflammatory responses. J Immunol, 192, 2326-38.

HARBER, K. J., DE GOEDE, K. E., VERBERK, S. G. S., MEINSTER, E., DE VRIES, H. E., VAN WEEGHEL, M., DE WINTHER, M. P. J. \& VAN DEN BOSSCHE, J. 2020. Succinate Is an Inflammation-Induced Immunoregulatory Metabolite in Macrophages. Metabolites, 10.

HARTMANN, F. J. \& BENDALL, S. C. 2020. Immune monitoring using mass cytometry and related high-dimensional imaging approaches. Nat Rev Rheumatol, 16, 87-99.

HARTMANN, F. J., MRDJEN, D., MCCAFFREY, E., GLASS, D. R., GREENWALD, N. F., BHARADWAJ, A., KHAIR, Z., VERBERK, S. G. S., BARANSKI, A., BASKAR, R., GRAF, W., VAN VALEN, D., VAN DEN BOSSCHE, J., ANGELO, M. \& BENDALL, S. 
C. 2021. Single-cell metabolic profiling of human cytotoxic T cells. Nat Biotechnol, 39, 186-197.

HUANG, S. C., EVERTS, B., IVANOVA, Y., O'SULLIVAN, D., NASCIMENTO, M., SMITH, A. M., BEATTY, W., LOVE-GREGORY, L., LAM, W. Y., O'NEILL, C. M., YAN, C., DU, H., ABUMRAD, N. A., URBAN, J. F., JR., ARTYOMOV, M. N., PEARCE, E. L. \& PEARCE, E. J. 2014a. Cell-intrinsic lysosomal lipolysis is essential for alternative activation of macrophages. Nat Immunol, 15, 846-55.

HUANG, S. C., SMITH, A. M., EVERTS, B., COLONNA, M., PEARCE, E. L., SCHILLING, J. D. \& PEARCE, E. J. 2016. Metabolic Reprogramming Mediated by the mTORC2IRF4 Signaling Axis Is Essential for Macrophage Alternative Activation. Immunity, 45, 817-830.

HUANG, Y. L., MORALES-ROSADO, J., RAY, J., MYERS, T. G., KHO, T., LU, M. \& MUNFORD, R. S. 2014b. Toll-like receptor agonists promote prolonged triglyceride storage in macrophages. J Biol Chem, 289, 3001-12.

JANSSEN, J. J. E., LAGERWAARD, B., BUNSCHOTEN, A., SAVELKOUL, H. F. J., VAN NEERVEN, R. J. J., KEIJER, J. \& DE BOER, V. C. J. 2021. Novel standardized method for extracellular flux analysis of oxidative and glycolytic metabolism in peripheral blood mononuclear cells. Sci Rep, 11, 1662.

JHA, A. K., HUANG, S. C., SERGUSHICHEV, A., LAMPROPOULOU, V., IVANOVA, Y., LOGINICHEVA, E., CHMIELEWSKI, K., STEWART, K. M., ASHALL, J., EVERTS, B., PEARCE, E. J., DRIGGERS, E. M. \& ARTYOMOV, M. N. 2015. Network integration of parallel metabolic and transcriptional data reveals metabolic modules that regulate macrophage polarization. Immunity, 42, 419-30.

KEREN, L., BOSSE, M., THOMPSON, S., RISOM, T., VIJAYARAGAVAN, K., MCCAFFREY, E., MARQUEZ, D., ANGOSHTARI, R., GREENWALD, N. F., FIENBERG, H., WANG, J., KAMBHAM, N., KIRKWOOD, D., NOLAN, G., MONTINE, T. J., GALLI, S. J., WEST, R., BENDALL, S. C. \& ANGELO, M. 2019. MIBI-TOF: A multiplexed imaging platform relates cellular phenotypes and tissue structure. Sci Adv, 5, eaax5851.

LAMPROPOULOU, V., SERGUSHICHEV, A., BAMBOUSKOVA, M., NAIR, S., VINCENT, E. E., LOGINICHEVA, E., CERVANTES-BARRAGAN, L., MA, X., HUANG, S. C., GRISS, T., WEINHEIMER, C. J., KHADER, S., RANDOLPH, G. J., PEARCE, E. J., JONES, R. G., DIWAN, A., DIAMOND, M. S. \& ARTYOMOV, M. N. 2016. Itaconate Links Inhibition of Succinate Dehydrogenase with Macrophage Metabolic Remodeling and Regulation of Inflammation. Cell Metab, 24, 158-66.

LAUTERBACH, M. A., HANKE, J. E., SEREFIDOU, M., MANGAN, M. S. J., KOLBE, C. C., HESS, T., ROTHE, M., KAISER, R., HOSS, F., GEHLEN, J., ENGELS, G., KREUTZENBECK, M., SCHMIDT, S. V., CHRIST, A., IMHOF, A., HILLER, K. \& LATZ, E. 2019. Toll-like Receptor Signaling Rewires Macrophage Metabolism and Promotes Histone Acetylation via ATP-Citrate Lyase. Immunity, 51, 997-1011 e7.

LEVINE, L. S., HIAM-GALVEZ, K. J., MARQUEZ, D. M., TENVOOREN, I., MADDEN, M. Z., CONTRERAS, D. C., DAHUNSI, D. O., IRISH, J. M., OLUWOLE, O. O., RATHMELL, J. C. \& SPITZER, M. H. 2021. Single-cell analysis by mass cytometry reveals metabolic states of early-activated CD8(+) T cells during the primary immune response. Immunity, 54, 829-844 e5.

LIM, A. R., RATHMELL, W. K. \& RATHMELL, J. C. 2020. The tumor microenvironment as a metabolic barrier to effector T cells and immunotherapy. Elife, 9, e55185.

LITTLE, A. C., KOVALENKO, I., GOO, L. E., HONG, H. S., KERK, S. A., YATES, J. A., PUROHIT, V., LOMBARD, D. B., MERAJVER, S. D. \& LYSSIOTIS, C. A. 2020. Highcontent fluorescence imaging with the metabolic flux assay reveals insights into mitochondrial properties and functions. Commun Biol, 3, 271.

LLUFRIO, E. M., WANG, L., NASER, F. J. \& PATTI, G. J. 2018. Sorting cells alters their redox state and cellular metabolome. Redox Biol, 16, 381-387.

MAKOWSKI, L., CHAIB, M. \& RATHMELL, J. C. 2020. Immunometabolism: From basic mechanisms to translation. Immunological reviews, 295, 5-14. 
MILLER, A., NAGY, C., KNAPP, B., LAENGLE, J., PONWEISER, E., GROEGER, M., STARKL, P., BERGMANN, M., WAGNER, O. \& HASCHEMI, A. 2017. Exploring Metabolic Configurations of Single Cells within Complex Tissue Microenvironments. Cell Metab, 26, 788-800 e6.

MILLS, E. L., KELLY, B., LOGAN, A., COSTA, A. S. H., VARMA, M., BRYANT, C. E., TOURLOMOUSIS, P., DABRITZ, J. H. M., GOTTLIEB, E., LATORRE, I., CORR, S. C., MCMANUS, G., RYAN, D., JACOBS, H. T., SZIBOR, M., XAVIER, R. J., BRAUN, T., FREZZA, C., MURPHY, M. P. \& O'NEILL, L. A. 2016. Succinate Dehydrogenase Supports Metabolic Repurposing of Mitochondria to Drive Inflammatory Macrophages. Cell, 167, 457-470 e13.

MOOKERJEE, S. A., GERENCSER, A. A., NICHOLLS, D. G. \& BRAND, M. D. 2017. Quantifying intracellular rates of glycolytic and oxidative ATP production and consumption using extracellular flux measurements. J Biol Chem, 292, 7189-7207.

MURPHY, M. P. \& O'NEILL, L. A. J. 2020. How should we talk about metabolism? Nat Immunol, 21, 713-715.

MURRAY, P. J., ALLEN, J. E., BISWAS, S. K., FISHER, E. A., GILROY, D. W., GOERDT, S., GORDON, S., HAMILTON, J. A., IVASHKIV, L. B., LAWRENCE, T., LOCATI, M., MANTOVANI, A., MARTINEZ, F. O., MEGE, J. L., MOSSER, D. M., NATOLI, G., SAEIJ, J. P., SCHULTZE, J. L., SHIREY, K. A., SICA, A., SUTTLES, J., UDALOVA, I., VAN GINDERACHTER, J. A., VOGEL, S. N. \& WYNN, T. A. 2014. Macrophage activation and polarization: nomenclature and experimental guidelines. Immunity, 41, 14-20.

NAMGALADZE, D. \& BRUNE, B. 2014. Fatty acid oxidation is dispensable for human macrophage IL-4-induced polarization. Biochim Biophys Acta, 1841, 1329-35.

NOMURA, M., LIU, J., ROVIRA, II, GONZALEZ-HURTADO, E., LEE, J., WOLFGANG, M. J. \& FINKEL, T. 2016. Fatty acid oxidation in macrophage polarization. Nat Immunol, 17, 216-7.

PALMER, A., PHAPALE, P., CHERNYAVSKY, I., LAVIGNE, R., FAY, D., TARASOV, A., KOVALEV, V., FUCHSER, J., NIKOLENKO, S., PINEAU, C., BECKER, M. \& ALEXANDROV, T. 2017. FDR-controlled metabolite annotation for high-resolution imaging mass spectrometry. Nat Methods, 14, 57-60.

RATTIGAN, K. M., POUNTAIN, A. W., REGNAULT, C., ACHCAR, F., VINCENT, I. M., GOODYEAR, C. S. \& BARRETT, M. P. 2018. Metabolomic profiling of macrophages determines the discrete metabolomic signature and metabolomic interactome triggered by polarising immune stimuli. PLoS One, 13, e0194126.

REALES-CALDERON, J. A., AGUILERA-MONTILLA, N., CORBI, A. L., MOLERO, G. \& GIL, C. 2014. Proteomic characterization of human proinflammatory M1 and antiinflammatory M2 macrophages and their response to Candida albicans. Proteomics, $14,1503-18$.

RODRÍGUEZ-PRADOS, J.-C., TRAVÉS, P. G., CUENCA, J., RICO, D., ARAGONÉS, J., MARTÍN-SANZ, P., CASCANTE, M. \& BOSCÁ, L. 2010. Substrate Fate in Activated Macrophages: A Comparison between Innate, Classic, and Alternative Activation. The Journal of Immunology, 185, 605-614.

SALABEI, J. K., GIBB, A. A. \& HILL, B. G. 2014. Comprehensive measurement of respiratory activity in permeabilized cells using extracellular flux analysis. Nat Protoc, 9, 421-38.

SCHNEEMANN, M., SCHOEDON, G., HOFER, S., BLAU, N., GUERRERO, L. \& SCHAFFNER, A. 1993. Nitric oxide synthase is not a constituent of the antimicrobial armature of human mononuclear phagocytes. J Infect Dis, 167, 1358-63.

SINCLAIR, L. V., BARTHELEMY, C. \& CANTRELL, D. A. 2020. Single Cell Glucose Uptake Assays: A Cautionary Tale. Immunometabolism, 2, e200029.

THOMAS, A. C. \& MATTILA, J. T. 2014. "Of mice and men": arginine metabolism in macrophages. Front Immunol, 5, 479.

VAN DEN BOSSCHE, J., BAARDMAN, J. \& DE WINTHER, M. P. 2015. Metabolic characterization of polarized M1 and M2 bone marrow-derived macrophages using 
real-time extracellular flux analysis. JoVE (Journal of Visualized Experiments), e53424.

VAN DEN BOSSCHE, J., BAARDMAN, J., OTTO, N. A., VAN DER VELDEN, S., NEELE, A. E., VAN DEN BERG, S. M., LUQUE-MARTIN, R., CHEN, H. J., BOSHUIZEN, M. C., AHMED, M., HOEKSEMA, M. A., DE VOS, A. F. \& DE WINTHER, M. P. 2016. Mitochondrial Dysfunction Prevents Repolarization of Inflammatory Macrophages. Cell Rep, 17, 684-696.

VAN DEN BOSSCHE, J., O'NEILL, L. A. \& MENON, D. 2017. Macrophage Immunometabolism: Where Are We (Going)? Trends Immunol, 38, 395-406.

VAN DEN BOSSCHE, J. \& VAN DER WINDT, G. J. W. 2018. Fatty Acid Oxidation in Macrophages and T Cells: Time for Reassessment? Cell Metab, 28, 538-540.

VAN DER WINDT, G. J., EVERTS, B., CHANG, C. H., CURTIS, J. D., FREITAS, T. C., AMIEL, E., PEARCE, E. J. \& PEARCE, E. L. 2012. Mitochondrial respiratory capacity is a critical regulator of CD8+ T cell memory development. Immunity, 36, 68-78.

VAN GASSEN, S., CALLEBAUT, B., VAN HELDEN, M. J., LAMBRECHT, B. N., DEMEESTER, P., DHAENE, T. \& SAEYS, Y. 2015. FlowSOM: Using self-organizing maps for visualization and interpretation of cytometry data. Cytometry A, 87, 636-45.

VATS, D., MUKUNDAN, L., ODEGAARD, J. I., ZHANG, L., SMITH, K. L., MOREL, C. R., WAGNER, R. A., GREAVES, D. R., MURRAY, P. J. \& CHAWLA, A. 2006. Oxidative metabolism and PGC-1beta attenuate macrophage-mediated inflammation. Cell Metab, 4, 13-24.

VIJAYAN, V., PRADHAN, P., BRAUD, L., FUCHS, H. R., GUELER, F., MOTTERLINI, R., FORESTI, R. \& IMMENSCHUH, S. 2019. Human and murine macrophages exhibit differential metabolic responses to lipopolysaccharide - A divergent role for glycolysis. Redox Biol, 22, 101147.

VOSS, K., HONG, H. S., BADER, J. E., SUGIURA, A., LYSSIOTIS, C. A. \& RATHMELL, J. C. 2021. A guide to interrogating immunometabolism. Nat Rev Immunol.

WANG, F., ZHANG, S., VUCKOVIC, I., JEON, R., LERMAN, A., FOLMES, C. D., DZEJA, P. P. \& HERRMANN, J. 2018. Glycolytic Stimulation Is Not a Requirement for M2 Macrophage Differentiation. Cell Metab, 28, 463-475 e4.

WYNN, T. A., CHAWLA, A. \& POLLARD, J. W. 2013. Macrophage biology in development, homeostasis and disease. Nature, 496, 445-55.

ZOROVA, L. D., POPKOV, V. A., PLOTNIKOV, E. Y., SILACHEV, D. N., PEVZNER, I. B., JANKAUSKAS, S. S., BABENKO, V. A., ZOROV, S. D., BALAKIREVA, A. V., JUHASZOVA, M., SOLLOTT, S. J. \& ZOROV, D. B. 2018. Mitochondrial membrane potential. Anal Biochem, 552, 50-59. 


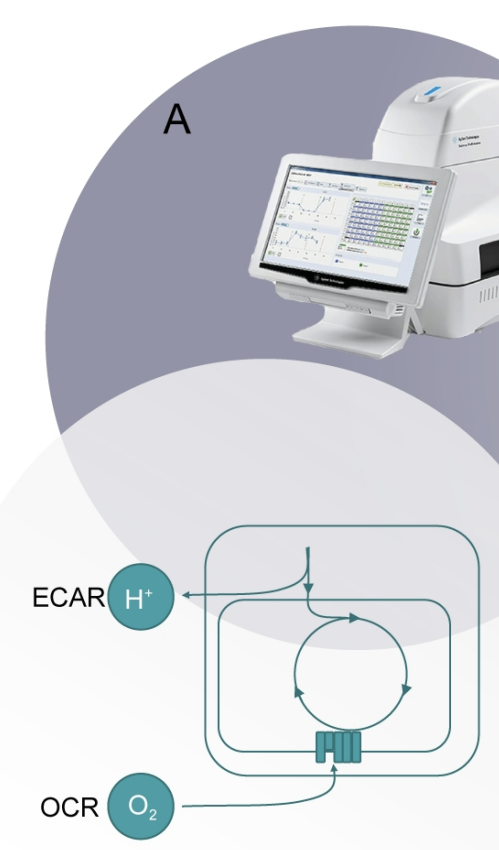

Inhibitors $\rightarrow \triangle \mathrm{ECAR}, \triangle \mathrm{OCR}$
B

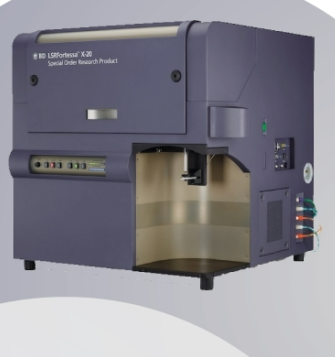

Glucose Fatty acids

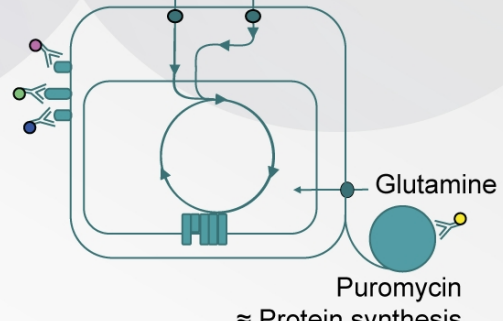

$\approx$ Protein synthesis

Inhibitors $\rightarrow \Delta$ protein synthesis

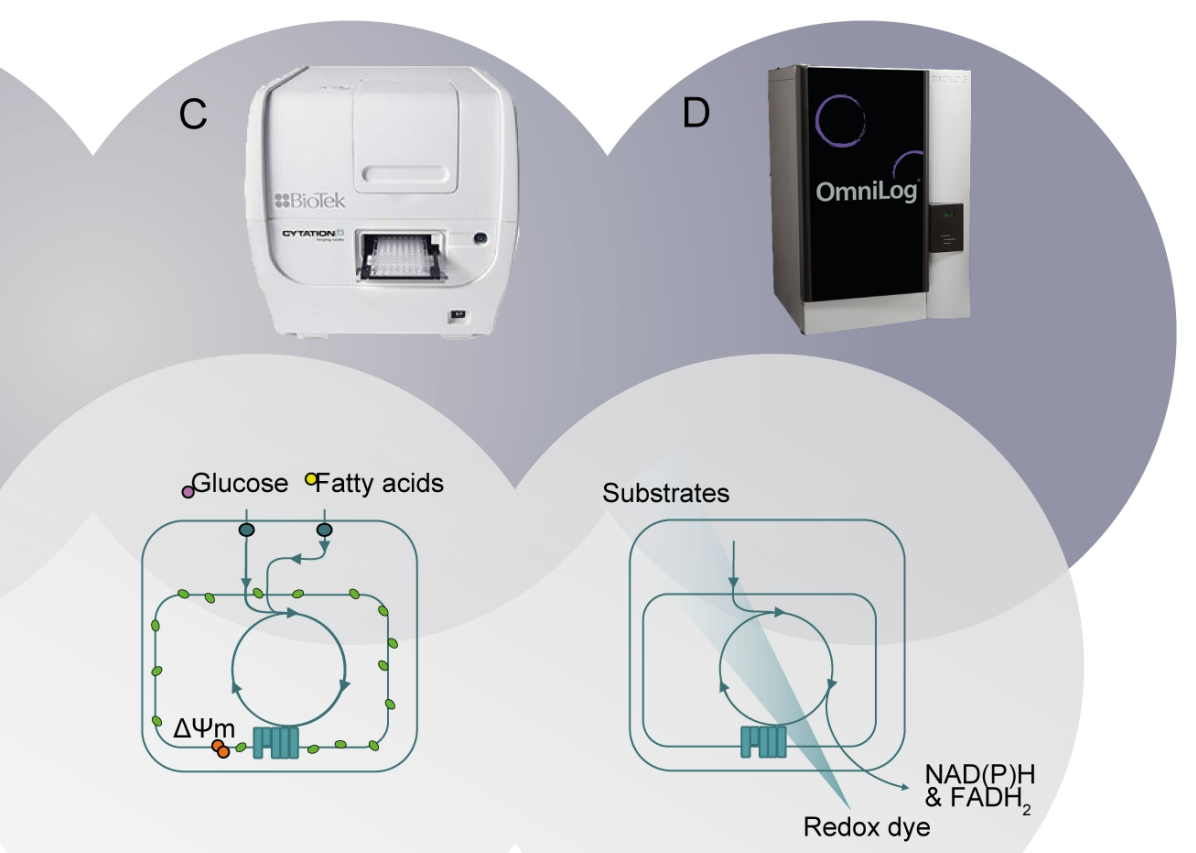

Redox dye 


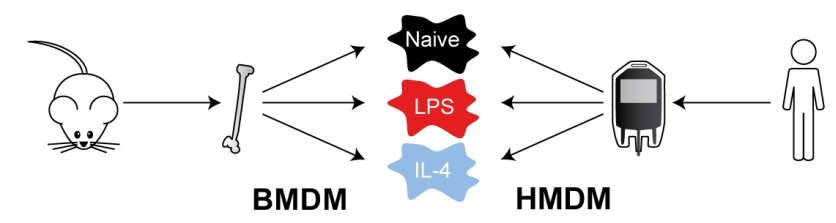

B
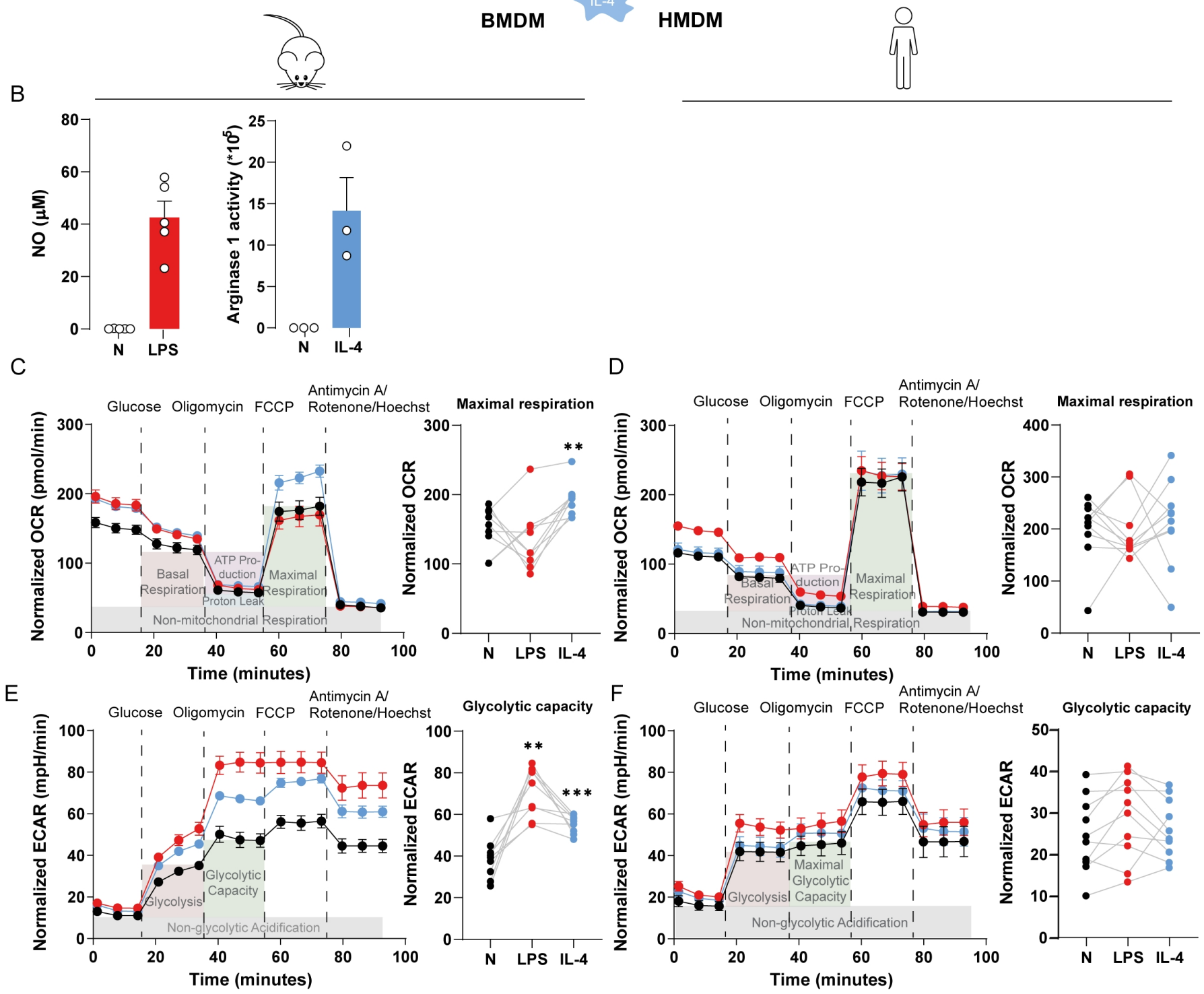

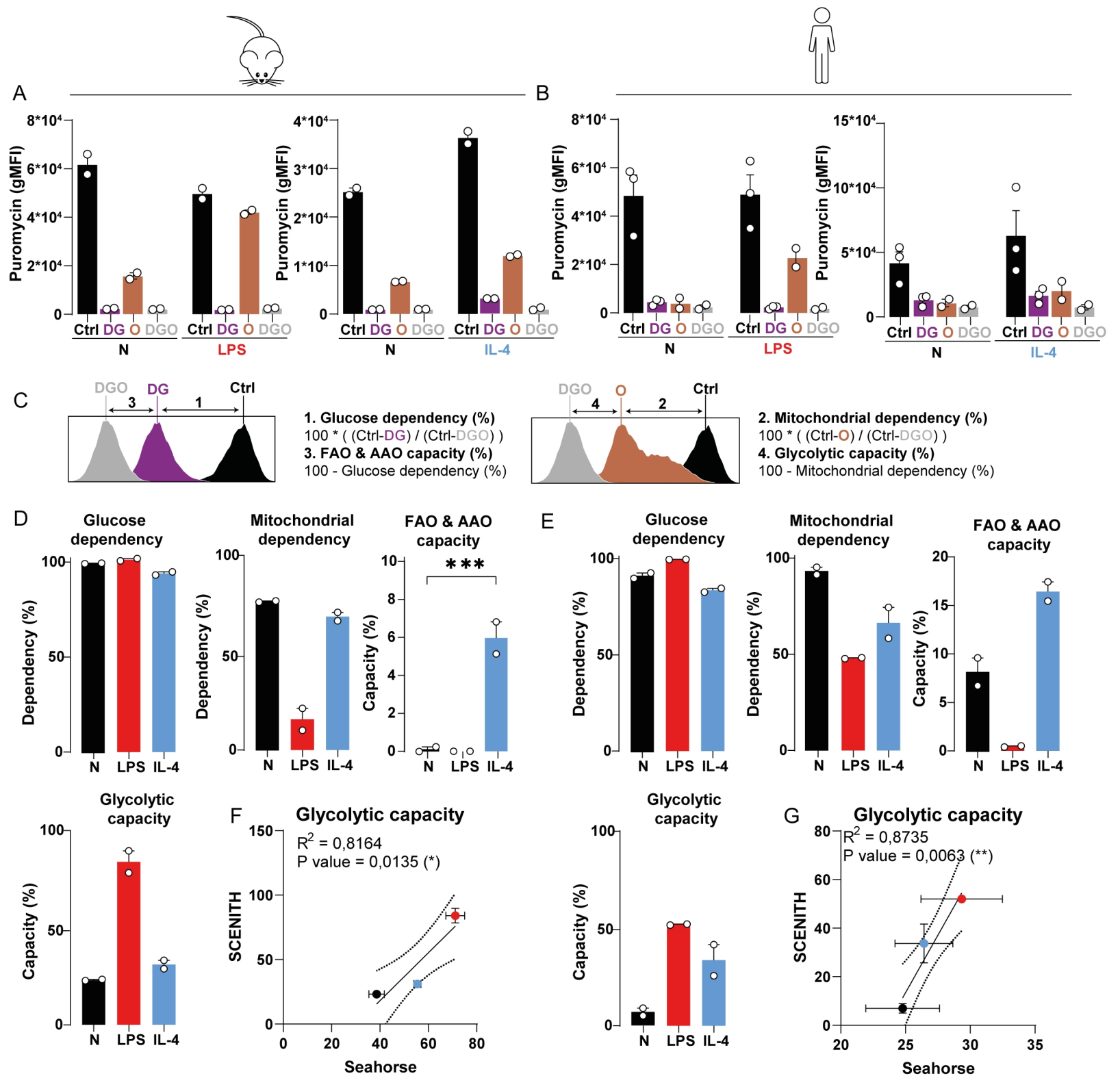
I
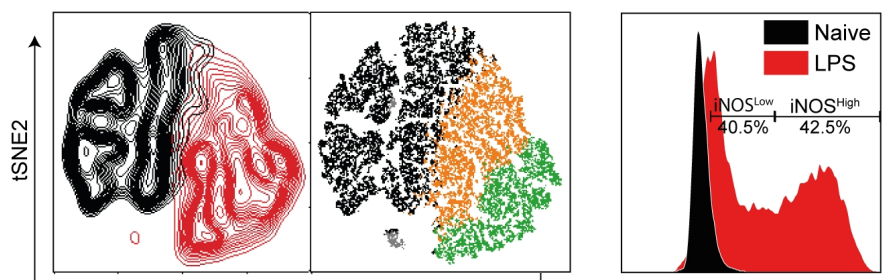

iNOS - PE
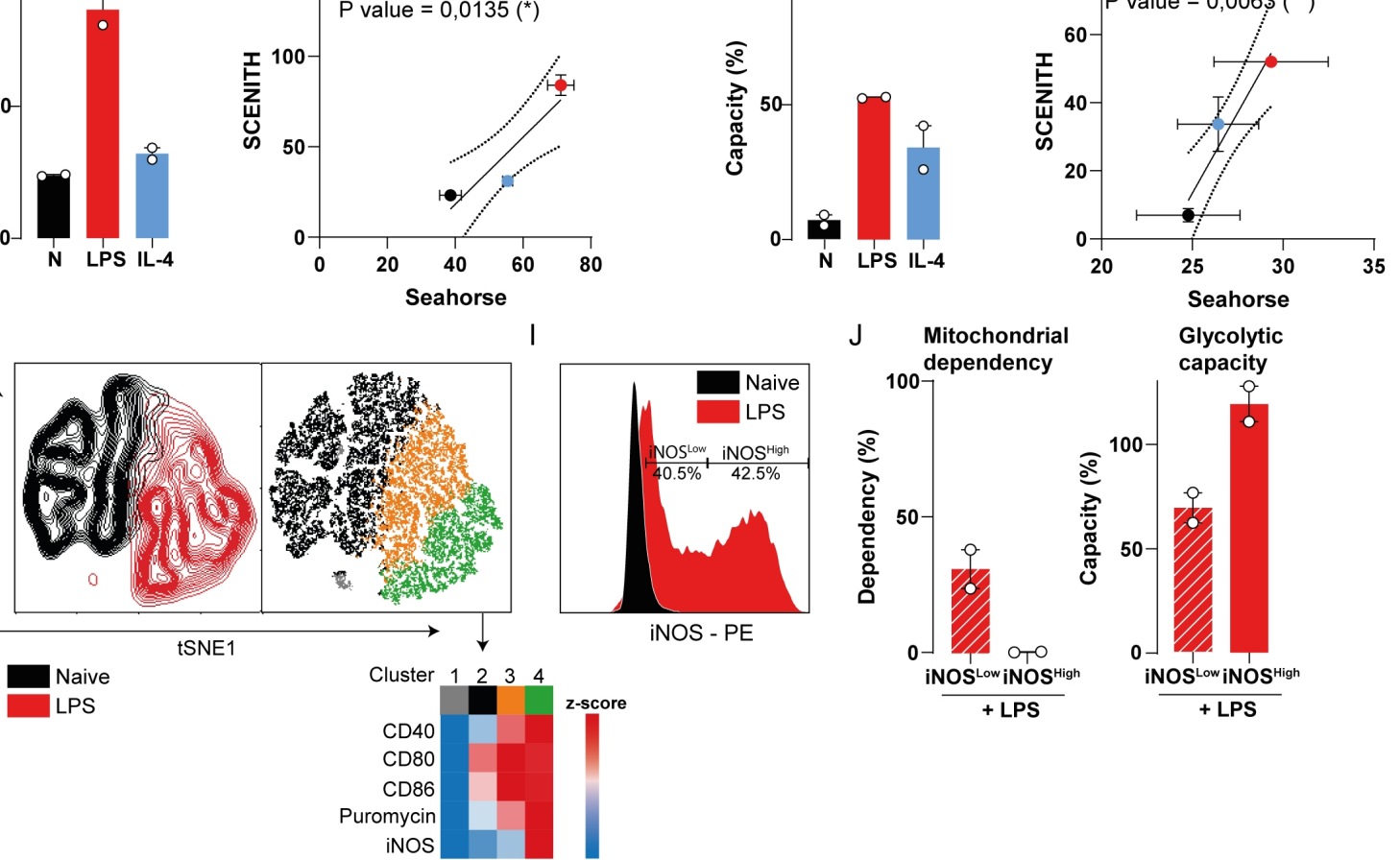
A
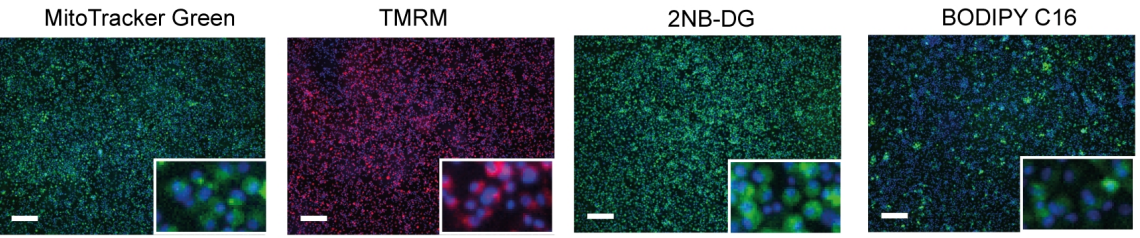

B
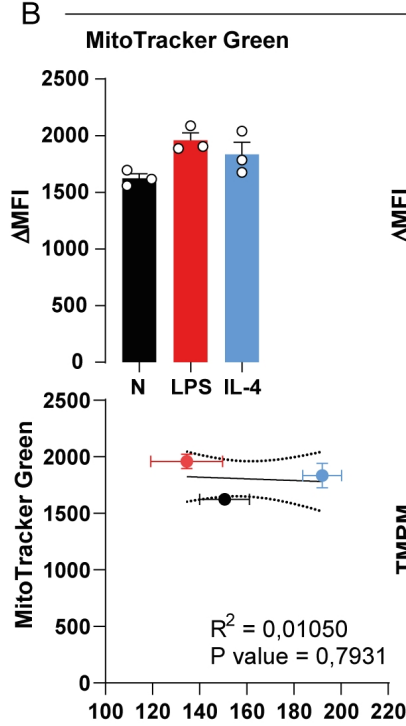

Max respiration

D
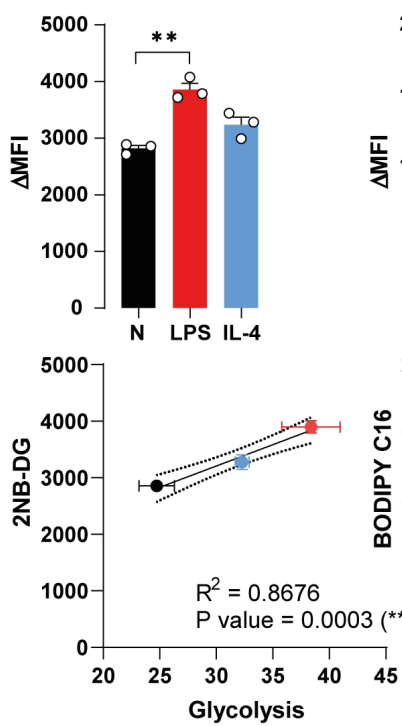
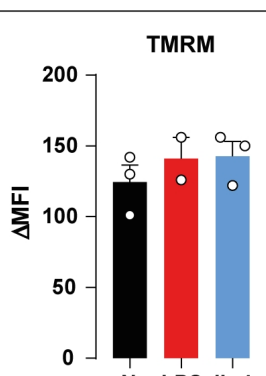

N LPS IL-4

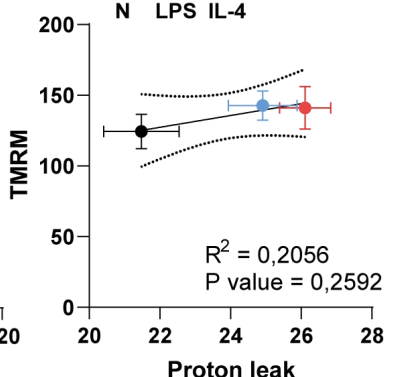

Proton leak
C
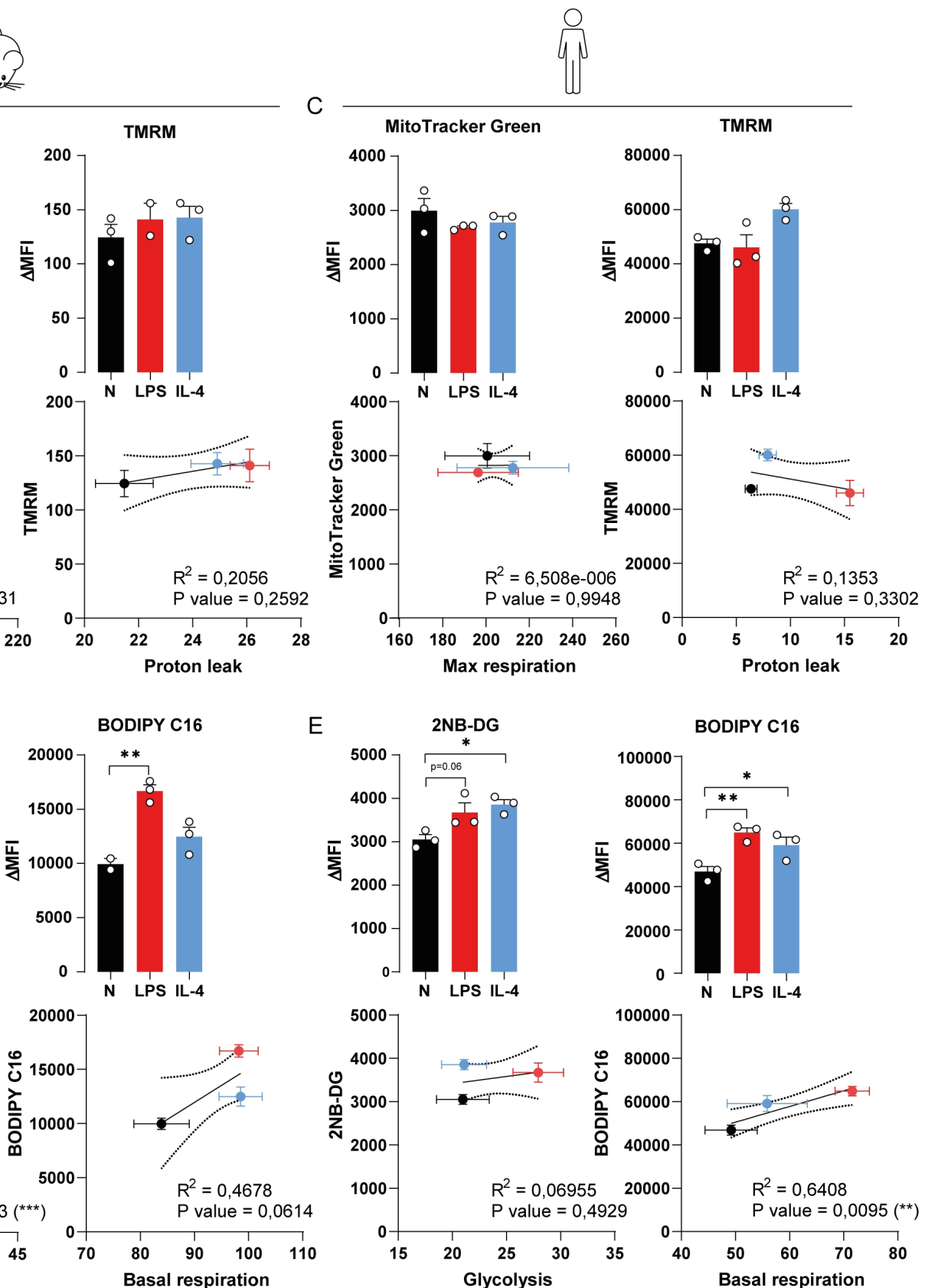

E
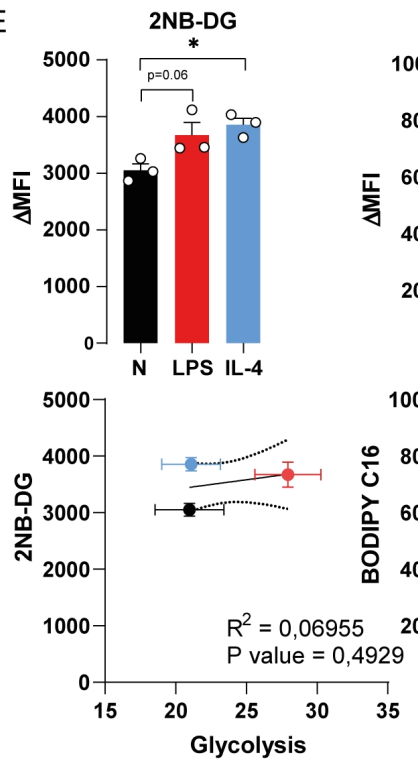

BODIPY C16
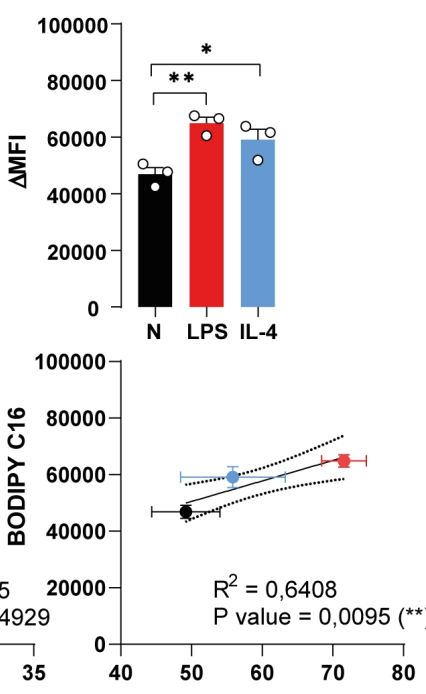


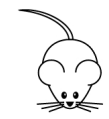

A
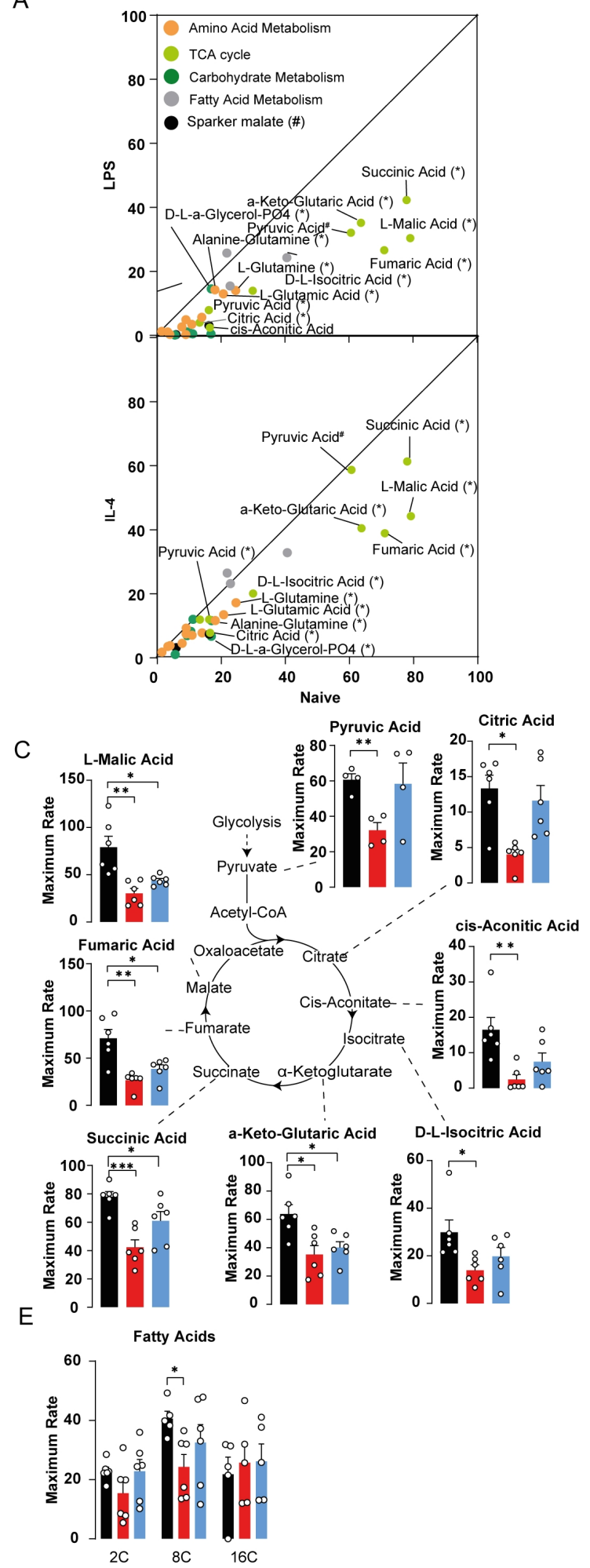

B
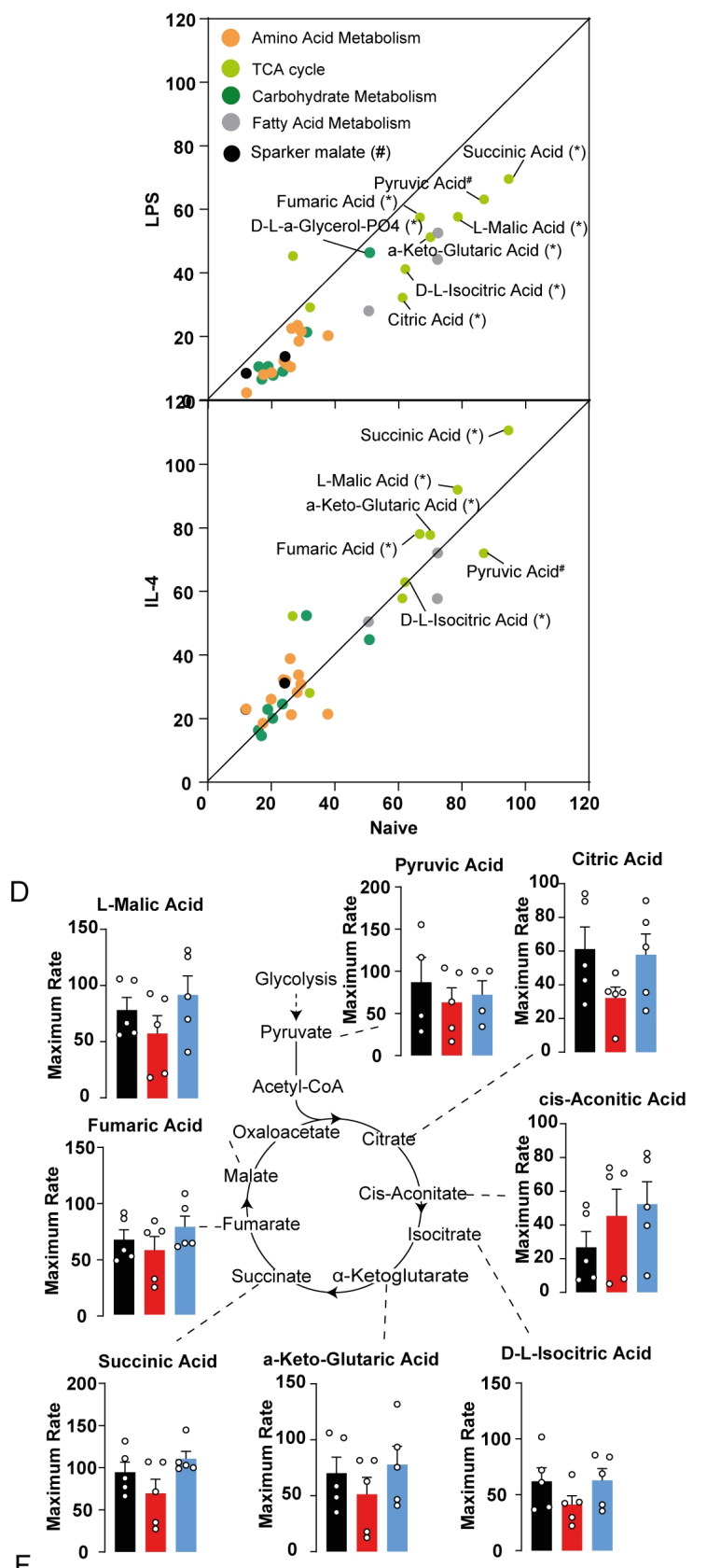

F Fatty Acids

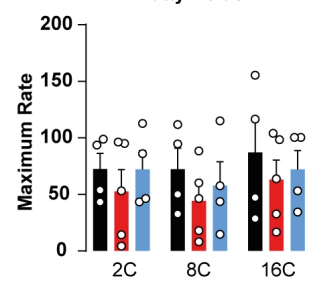




\section{Immunometabolic}

Metabolic prescreening toolbox

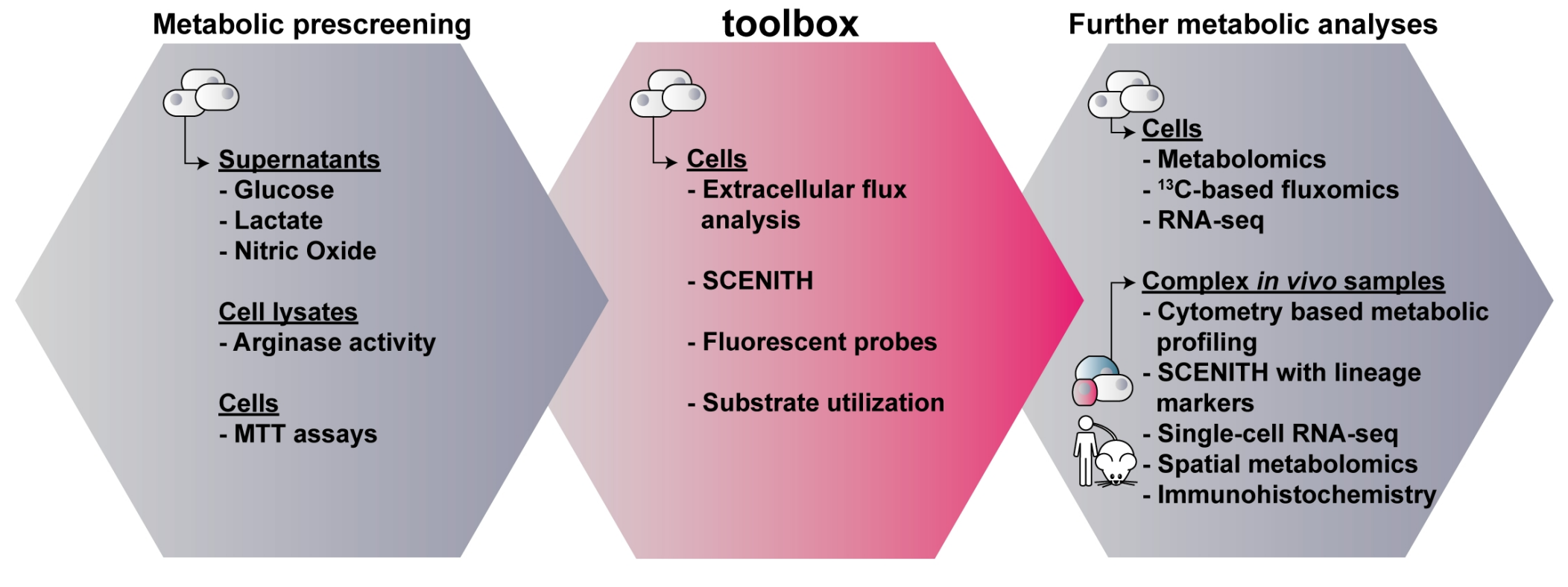




\section{Supplemental information}

Supplementary Table 1: List of antibodies used

\begin{tabular}{|c|c|c|c|c|}
\hline Target & Species & Clone & Fluorochrome & Company \\
\hline CD40 & $M$ & $3 / 23$ & APC & Biolegend \\
\hline CD80 & $\mathrm{M}$ & 16-10A1 & BV650 & Biolegend \\
\hline CD86 & $M$ & GL-1 & BV510 & Biolegend \\
\hline iNOS & $M$ & CXNFT & $\mathrm{PE}$ & eBioScience \\
\hline CD206 & $M$ & $\mathrm{C} 068 \mathrm{C} 2$ & BV605 & Biolegend \\
\hline CD301b & $M$ & URA-1 & PE-Cy7 & Biolegend \\
\hline CD273 & $\mathrm{M}$ & TY25 & PE-CF594 & Biolegend \\
\hline Arg1 & $M$ & A1exF5 & $\mathrm{PE}$ & eBioScience \\
\hline CD197 & $\mathrm{H}$ & G043H7 & FITC & Biolegend \\
\hline CD80 & $\mathrm{H}$ & 2D10 & PE-Cy7 & Biolegend \\
\hline CD200R & $\mathrm{H}$ & OX-108 & PE-CF594 & Biolegend \\
\hline CD206 & $\mathrm{H}$ & $15-2$ & APC & Biolegend \\
\hline Fixable Viability Dye & $\mathrm{n} / \mathrm{a}$ & $\mathrm{n} / \mathrm{a}$ & eFluor780 & eBioScience \\
\hline Puromycin & Rat & R4743L-E8 & AF488, AF647 & $\begin{array}{l}\text { SCENITH kit } \\
\text { www.scenith.com } \\
\begin{array}{l}\text { (Arguello et al., } \\
2020)\end{array}\end{array}$ \\
\hline
\end{tabular}



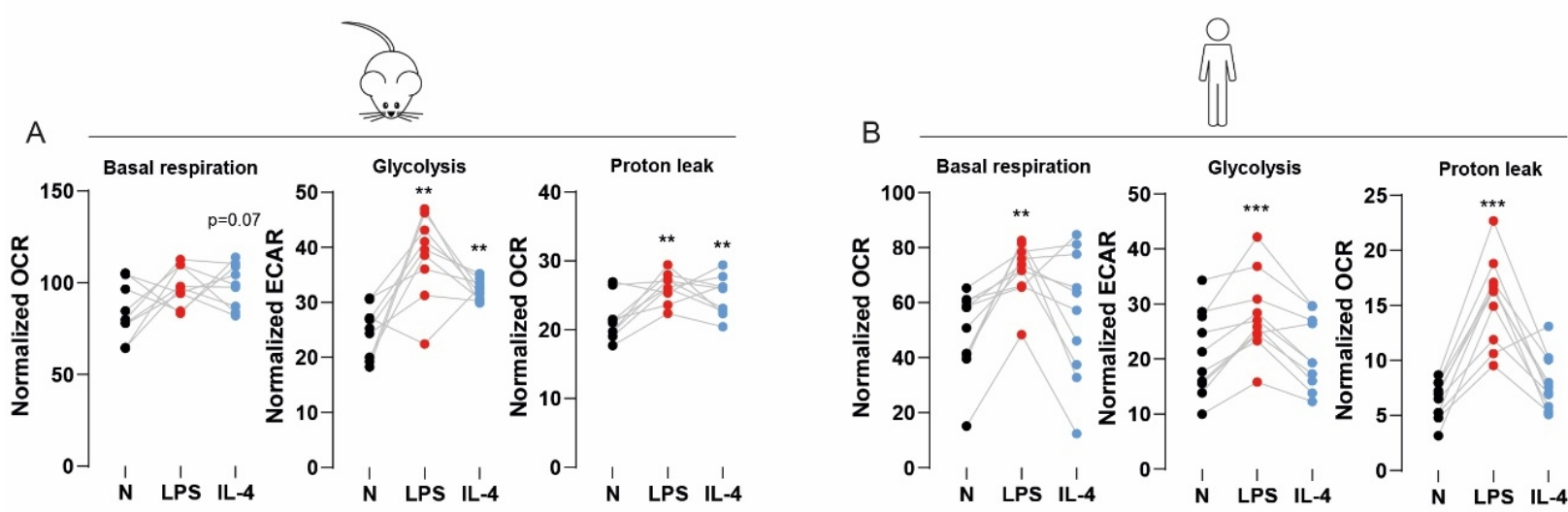

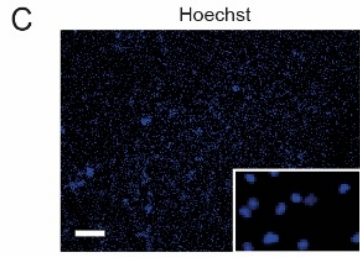

Brightfield + Hoechst

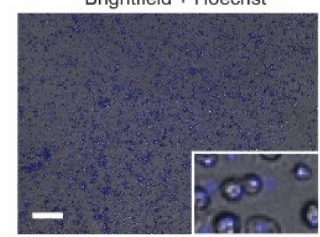

Glycolytic capacity Normalization per well

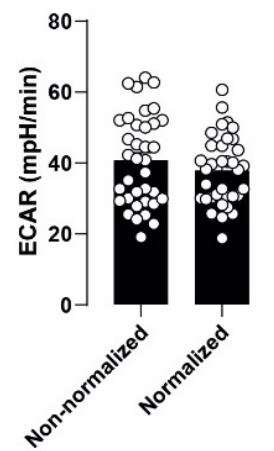

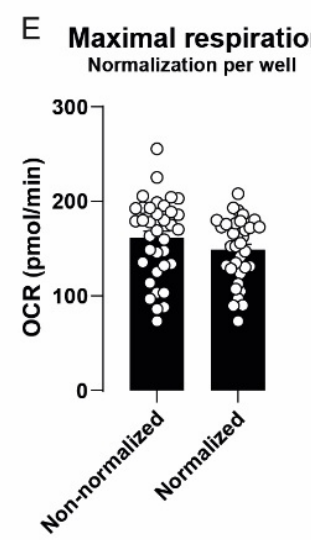

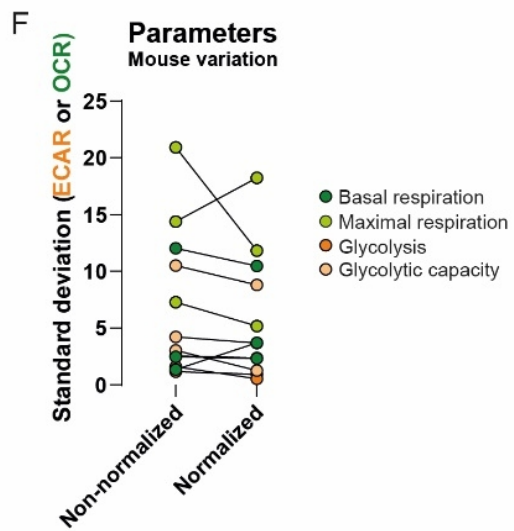

Supplementary Figure 1: Extracellular flux data were normalized with cell counts derived from cell permeable Hoechst staining. (A, B) Extracellular flux parameters extracted from OCR and ECAR data in BMDMs (A) and HMDMs (B) stimulated with LPS, IL-4 or left untreated. (C) Hoechst staining of cells in Seahorse plate for normalization, captured in fluorescent mode and overlaid with brightfield picture to validate recognition of cells rather than artifacts. Scale bar represents $200 \mu \mathrm{m}$. (D, E) Glycolytic capacity (D) and maximal respiration (E) of all measured wells before and after normalization with relative cell counts determined by Hoechst staining in 3 different experiments, each exploring unstimulated BMDMs from 3 different mice. $(F)$ Standard deviations of extracellular flux parameters from 3 mice measured within 1 experiment before and after normalization with relative cell count. Data are shown as median value per mouse $(N=9)$ or donor $(N=10)(A, B)$, as mean $\pm S E M(D, E)$ or as mean $S D$ per experiment $(\mathrm{N}=3$ experiments) $(\mathrm{F})$. 

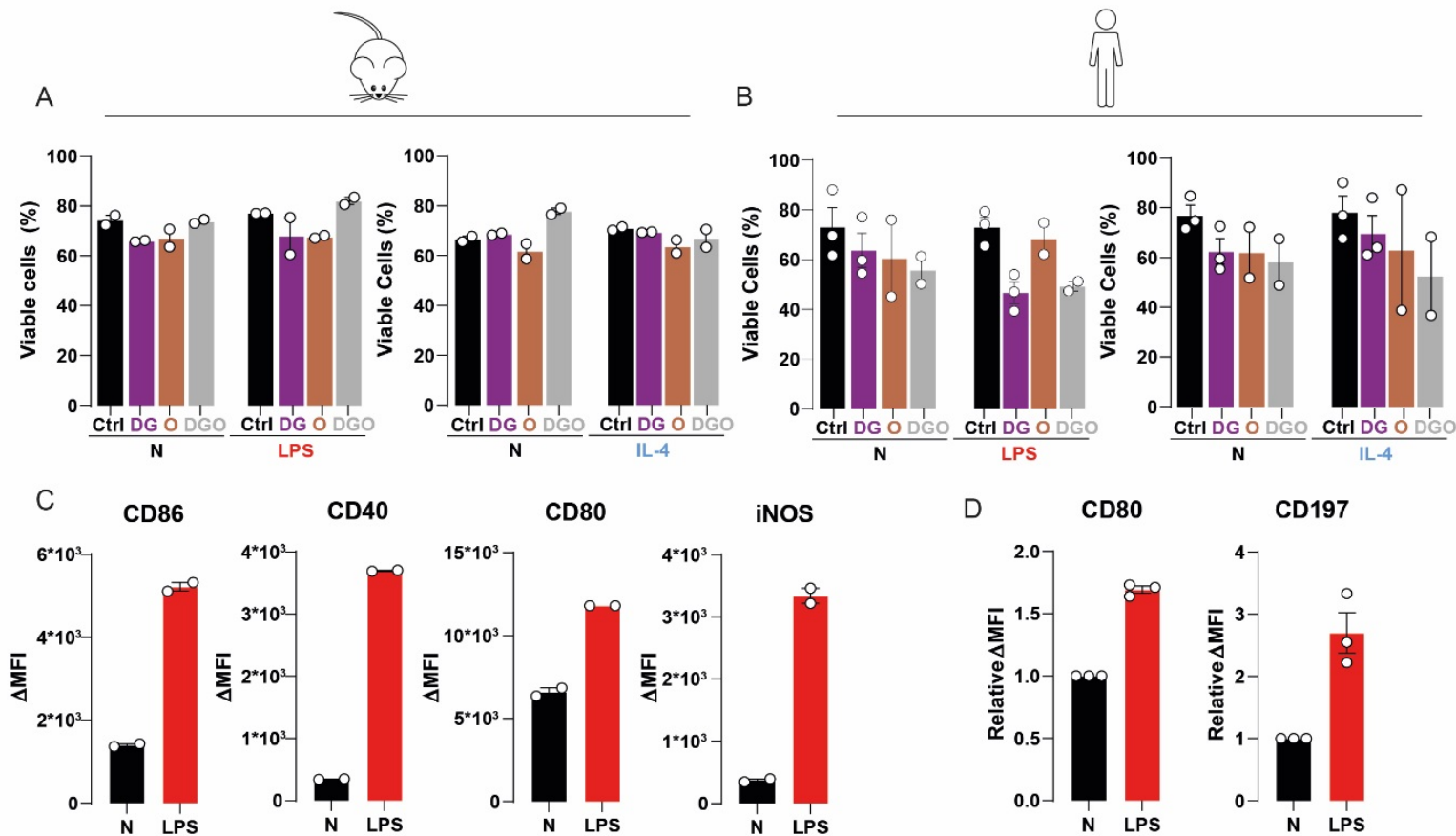

D $\quad$ CD80

CD197
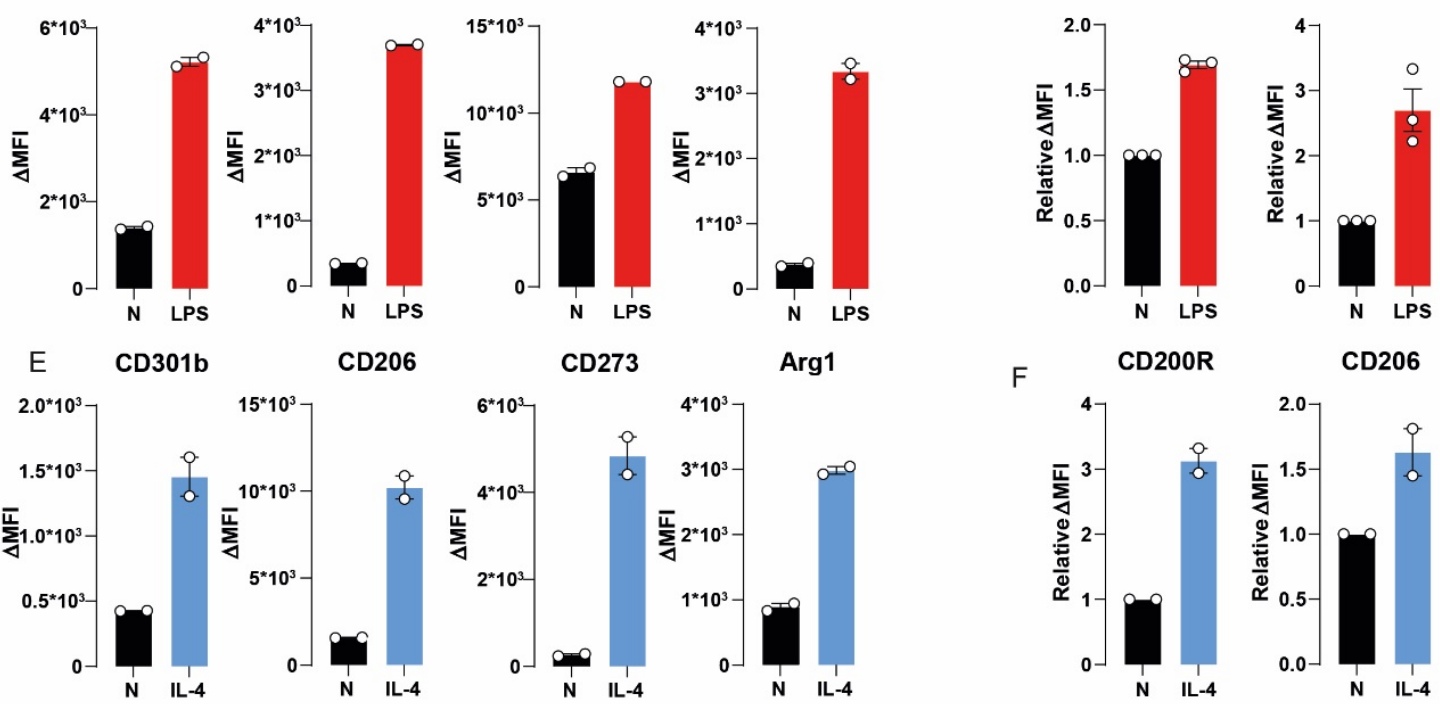

CD200R

CD206
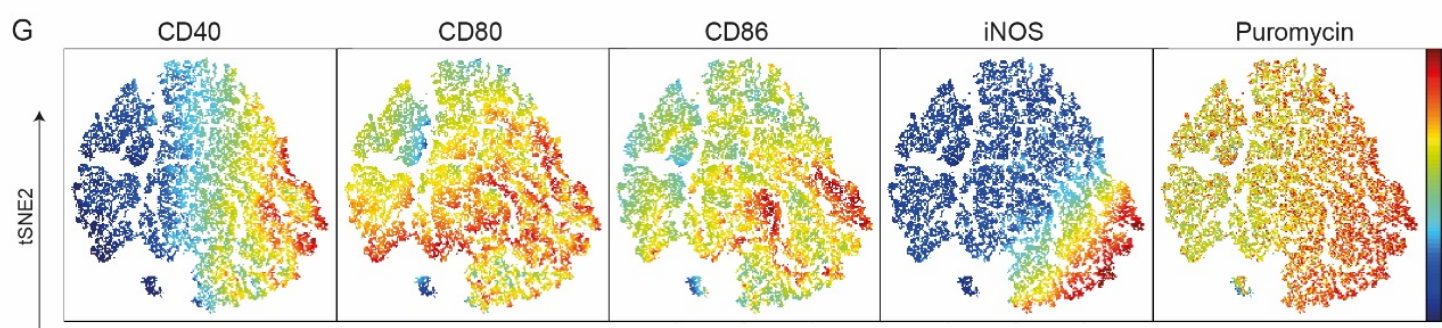

Supplementary Figure 2: Combination of SCENITH with macrophage surface markers confirms expected macrophage activation. (A, B) Viability of samples treated with different metabolic inhibitors during SCENITH protocol for comparison of LPS-activated and IL-4-activated with naïve BMDMs (A) and HMDMs (B). (C, D) Typical LPS-induced surface markers for BMDMs (C) and HMDMs (D). (E,F) Typical IL-4-induced surface markers for BMDMs (E) and HMDMs (F). (G) tSNE dimensionality reduction and marker expression of oligomycin-treated LPS-stimulated BMDMs. Data are shown as mean \pm SEM. Each dot marks a separate mouse $(\mathrm{N}=2)$ or donor $(\mathrm{N}=2-3)$. $\triangle \mathrm{MFI}$ was calculated as MFI (median fluorescent intensity) of sample - MFI of unstained control. 
A

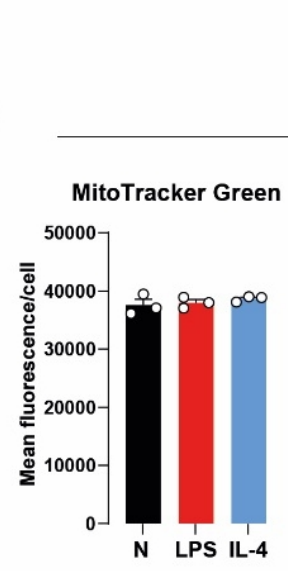

2NB-DG

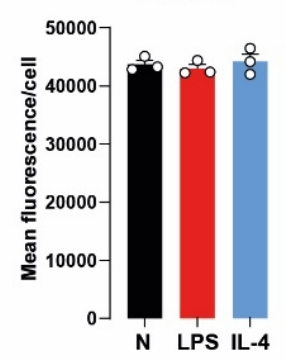

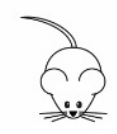

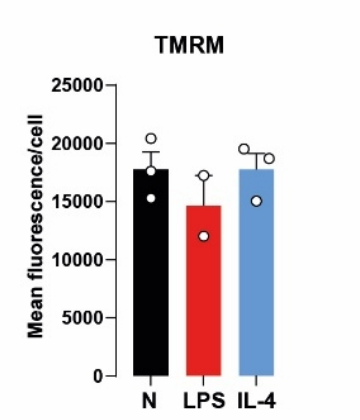

BODIPY C16

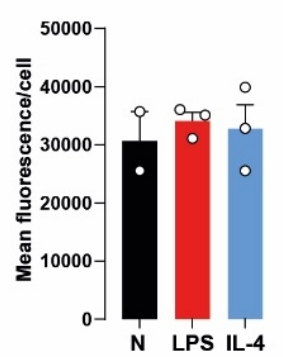

B

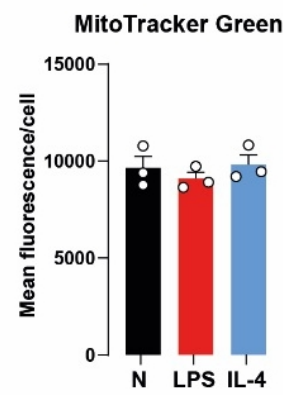

2NB-DG

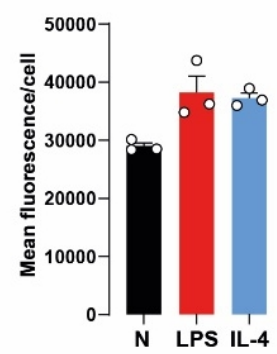

TMRM

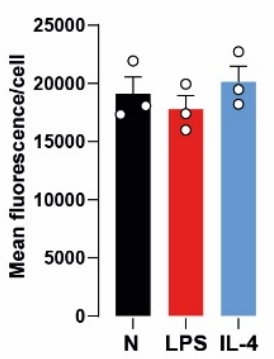

BODIPY C16

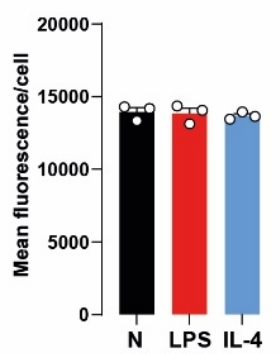

Supplementary Figure 3: Imaging of fluorescent probe uptake with a multi-mode reader does not reveal differences between activation states. $(A, B)$ Fluorescent imaging of MitoTracker Green, TMRM, 2NB-DG and BODIPY C16 with a multi-mode reader for BMDMs (A) and HMDMs (B). Data are shown as mean \pm SEM. Each dot marks a separate mouse $(\mathrm{N}=2-3)$ or donor $(\mathrm{N}=3)$. $\Delta \mathrm{MFI}$ was calculated as MFI (median fluorescent intensity) of sample - MFI of unstained control. 


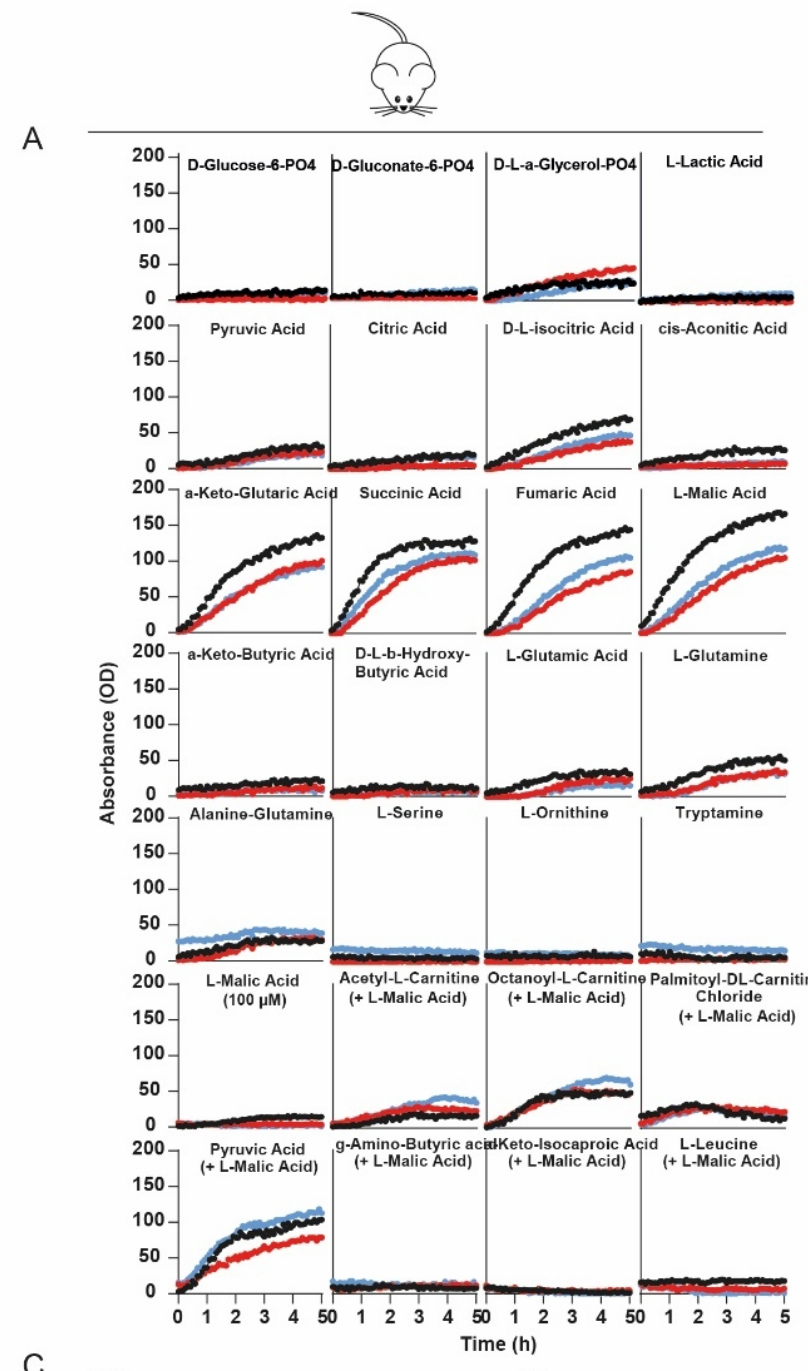

C
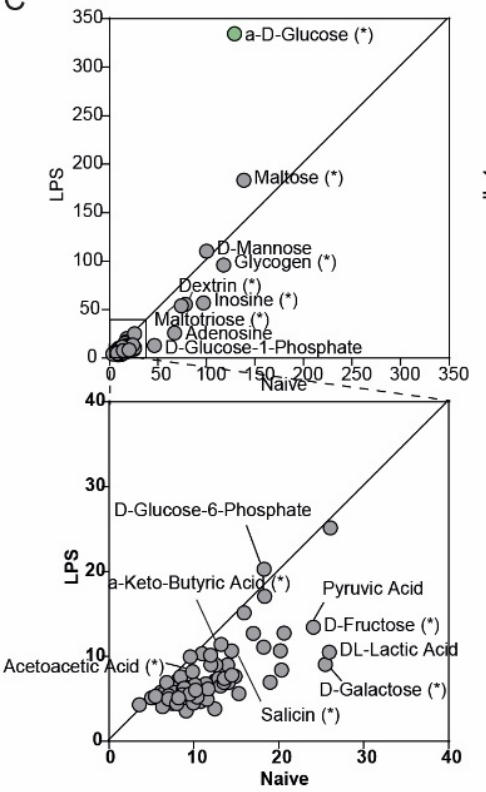
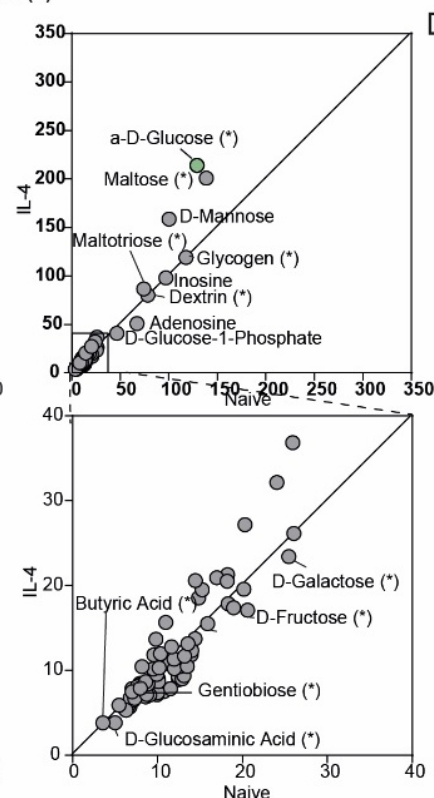
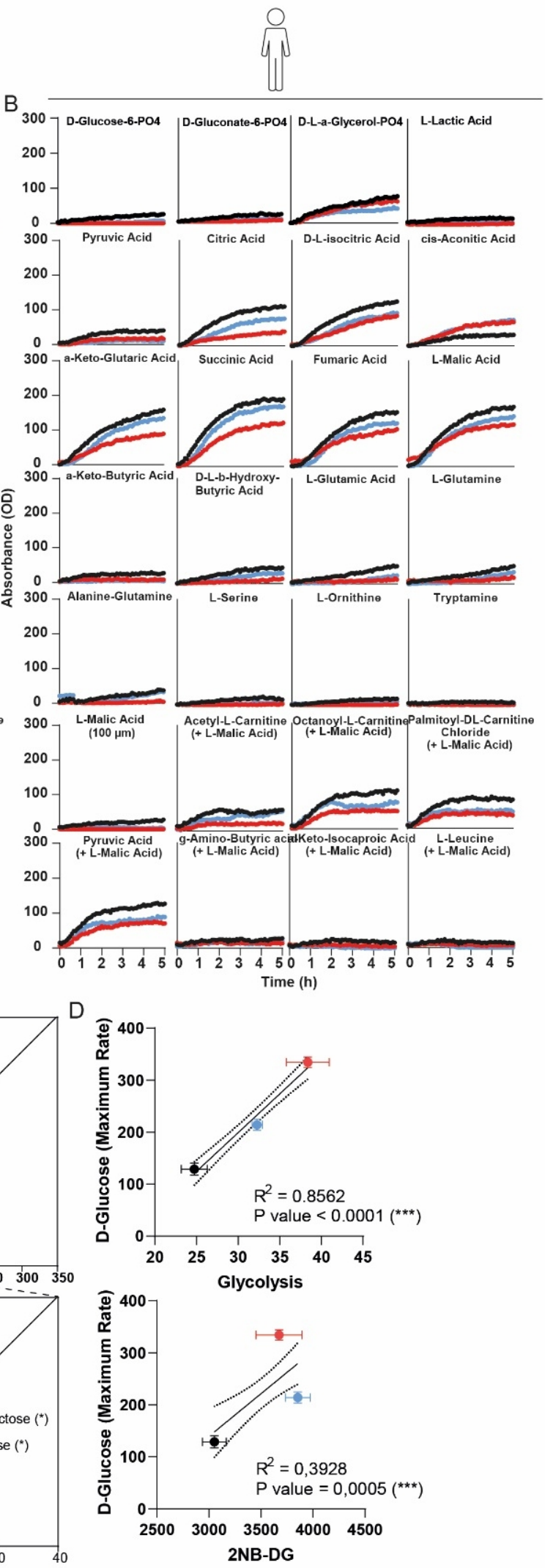

Supplementary Figure 4: Kinetic rates of Mito-S1 plates and analysis of substrate oxidation by PM-M01 plates. $(A, B)$ Kinetic rates of substrate usage by permeabilized BMDMs (A) and HMDMs (B). (C) Scatterplot comparisons of maximum rate in the timespan of 1-4h of substrate utilization by LPSand IL-4-activated versus naive BMDMs. (D) Correlation of maximum D-glucose uptake as measured by Biolog PM-M01 plates and glycolysis as measured by extracellular flux analysis and 2NB-DG as measured by flow cytometry. 